\title{
Density-functional theory with screened van der Waals interactions applied to atomic and molecular adsorbates on close-packed and non-close-packed surfaces
}

\author{
Victor G. Ruiz, Wei Liu, and Alexandre Tkatchenko \\ Fritz-Haber-Institut der Max-Planck-Gesellschaft, Faradayweg 4-6, 14195, Berlin, Germany \\ (Received 23 February 2015; revised manuscript received 15 October 2015; published 15 January 2016)
}

\begin{abstract}
Modeling the adsorption of atoms and molecules on surfaces requires efficient electronic-structure methods that are able to capture both covalent and noncovalent interactions in a reliable manner. In order to tackle this problem, we have developed a method within density-functional theory (DFT) to model screened van der Waals interactions ( $\mathrm{vdW}$ ) for atoms and molecules on surfaces (the so-called DFT $+\mathrm{vdW}^{\text {surf }}$ method). The relatively high accuracy of the DFT $+\mathrm{vdW}^{\text {surf }}$ method in the calculation of both adsorption distances and energies, as well as the high degree of its reliability across a wide range of adsorbates, indicates the importance of the collective electronic effects within the extended substrate for the calculation of the vdW energy tail. We examine in detail the theoretical background of the method and assess its performance for adsorption phenomena including the physisorption of Xe on selected close-packed transition metal surfaces and 3,4,9,10-perylene-tetracarboxylic acid dianhydride (PTCDA) on $\mathrm{Au}(111)$. We also address the performance of DFT+ $\mathrm{vdW}^{\text {surf }}$ in the case of non-close-packed surfaces by studying the adsorption of $\mathrm{Xe}$ on $\mathrm{Cu}(110)$ and the interfaces formed by the adsorption of a PTCDA monolayer on the $\operatorname{Ag}(111), \operatorname{Ag}(100)$, and $\operatorname{Ag}(110)$ surfaces. We conclude by discussing outstanding challenges in the modeling of vdW interactions for studying atomic and molecular adsorbates on inorganic substrates.
\end{abstract}

DOI: 10.1103/PhysRevB.93.035118

\section{INTRODUCTION}

Understanding the electronic properties of hybrid inorganic/organic systems (HIOS) has implications in both fundamental science and technology. In terms of basic science, these interfaces may lead to the emergence of collective effects that the isolated components forming the interface do not exhibit [1,2]. Among these effects, Kronik and Koch [1] mention the emergence of magnetic phenomena at the interface formed by nonmagnetic components (including closed-shell molecular layers), localization of electron-hole pairs at the interface, and electronic or transport properties of molecular ensembles that differ from those of the isolated molecule(s) (see references within Ref. [1]).

The eventual control of the function of HIOS has a substantial technological importance as well. Organic light-emitting diodes, organic thin-film transistors, and low cost and efficient organic solar cells are examples of emerging technologies based on organic thin films [1,3], which some of them are now reaching the consumer market [3]. Potential future applications also include organic memories and chemical sensors [1,3]. The performance and future design of these devices are clearly related to the electronic properties of the interface in which the interface geometry plays a fundamental role [4,5]. A balanced description of both the structural and electronic properties of these interfaces is thus critical for controlling their functionality.

The interplay of electron transfer processes, (covalent) hybridization of wave functions, van der Waals (vdW) interactions, and Pauli repulsion result in the interface properties that HIOS exhibit, including their interface structure. In particular, vdW forces play an essential part in the structure and stability of these systems [5-11]. Density-functional theory (DFT) has become the method of choice in the calculation of interfaces and adsorption phenomena due to its good compromise between accuracy and efficiency. Unfortunately, the modeling of vdW interactions in DFT is not an easy task as (semi)local and hybrid functionals used to approximate the exchange-correlation (xc) energy functional do not include them properly.

In this context, the role of $\mathrm{vdW}$ interactions in the binding between small molecules in the gas phase has been extensively studied and is fairly well understood. Unlike (semi)local functionals within DFT, the hierarchy of methods in quantum chemistry can describe $\mathrm{vdW}$ interactions properly. Recent years have also seen the development of several promising vdW-inclusive approaches in DFT. Methods such as DFT-D3 [12], vdW-DF2 [13], vdW-DF-type functionals with modified exchange [14], the DFT+XDM method [15,16], and the DFT $+v d W$ method [17] have shown to be quite accurate for intermolecular interactions (see, for example, Ref. [18] for a concise review of $\mathrm{vdW}$-inclusive methods in DFT). However, their application to HIOS is questionable due to either the absence or inaccuracy of the nonlocal (inhomogeneous) collective electron response of the extended surface in the vdW energy tail. This problem has been exemplified in previous publications for the case of the adsorption of 3,4,9,10-perylene-tetracarboxylic acid dianhydride $\left(\mathrm{C}_{24} \mathrm{H}_{8} \mathrm{O}_{6}\right.$, PTCDA) on coinage metal surfaces $[5,10,19-22]$ and in general for other adsorption systems [6-9,23,24].

The modeling of HIOS requires efficient methods that are able to describe a range of interactions in an accurate manner. In order to tackle this problem, we have developed the DFT $+\mathrm{vdW}^{\text {surf }}$ method [22] to calculate the adsorption properties of atoms and molecules on surfaces. This method combines the Tkatchenko-Scheffler (TS) DFT+vdW method [17] for intermolecular interactions with the LifshitzZaremba-Kohn (LZK) theory $[25,26]$ for the inclusion of the nonlocal collective response of the substrate surface in the vdW energy tail. Calculations using the DFT $+v_{d W} W^{\text {surf }}$ method have demonstrated that the inclusion of these collective effects, which effectively go beyond the atom-based pairwise description of $\mathrm{vdW}$ interactions, enables us to reliably describe the binding in many systems covering a wide range of 
interactions, including the adsorption of a Xe monolayer, of aromatic molecules (benzene and derivatives, naphthalene, anthracene, azobenzene, diindenoperylene, and olympicene and derivatives), $\mathrm{C}_{60}$, molecules including sulfur/oxygen such as thiophene, NTCDA, and PTCDA on several closepacked transition-metal surfaces [22,27-35]. A study of a $\mathrm{Cu}$-phthalocyanine film on a PTCDA monolayer adsorbed on $\mathrm{Ag}(111)$ has also been published recently [36].

In this work, we present a detailed description of the $\mathrm{DFT}+\mathrm{vdW} \mathrm{surf}^{\text {suthod }}$ methsess its performance on adsorption phenomena occurring at close-packed and non-closepacked surfaces. We start in Sec. II by reviewing the general theory of vdW interactions including the case of the atomsurface vdW interaction. We also discuss its relation to the $\mathrm{vdW}$ pairwise interactions and how this relation determines the development of the DFT $+\mathrm{vdW}^{\text {surf }}$ method. It is clear that the study of HIOS is central to our motivation. Nevertheless, it is also important to indicate that the adsorption of noble gases on metal surfaces are prototypical examples of adsorption phenomena where the main attractive forces are given by long-range $\mathrm{vdW}$ interactions. More interesting is the fact that they have been studied extensively in experiments [37-40] and theory [40-43]. Because of their status as benchmark systems for physisorption in vdW-inclusive DFT methods, we begin Sec. III by addressing the performance of the present method in the energetics and structure of the adsorption of Xe on selected close-packed transition-metal surfaces. As we progress in Sec. III, we also analyze the adsorption of a single PTCDA molecule on $\mathrm{Au}(111)$ as an example of an organic/inorganic interface. We take a similar approach to address the case of non-close-packed surfaces by first analyzing the adsorption of $\mathrm{Xe}$ on $\mathrm{Cu}(110)$ and comparing it to the case of $\mathrm{Cu}(111)$ and, as a final step, analyzing the adsorption of an organic adsorbate on a metallic surface with different orientations. For this, we take the interface formed by the adsorption of a PTCDA monolayer on the $\operatorname{Ag}(111), \operatorname{Ag}(100)$, and $\operatorname{Ag}(110)$ surfaces. Finally, in Sec. IV we give a brief summary and an outlook.

We mention the extensive study performed by Chen and co-workers [43] where they reported the performance of several vdW-inclusive methods within DFT on the adsorption of noble gases on metal surfaces which serves, together with this work, as a benchmark of DFT methods in physisorption phenomena. In the case of PTCDA on $\mathrm{Ag}(100)$ and $\mathrm{Ag}(110)$, we mention the theoretical and experimental studies reported in Refs. $[44,45]$ that were very helpful in the analysis that we present in this work.

\section{THEORY}

\section{A. The van der Waals interaction between polarizable fragments}

The vdW dispersion interactions result from correlated fluctuations between electrons. As a starting point, we consider the case of two neutral polarizable fragments $\mathcal{S}_{a}$ and $\mathcal{S}_{b}$ in the well-separated regime, where there is no wave-function overlap between fragments. The energy between the two fragments can be given (see, for example, Longuet-Higgins [46], Zaremba and Kohn [26]; Hartree atomic units used throughout) by

$$
\begin{aligned}
E_{\mathrm{vdW}}^{(2)}= & -\frac{1}{2 \pi} \int_{0}^{\infty} d \omega \int \ldots \int d \boldsymbol{r}_{a} d \boldsymbol{r}_{a}^{\prime} d \boldsymbol{r}_{b} d \boldsymbol{r}_{b}^{\prime} \\
& \times \chi_{a}\left(\boldsymbol{r}_{a}, \boldsymbol{r}_{a}^{\prime}, i \omega\right) v\left(\boldsymbol{r}_{a}^{\prime}, \boldsymbol{r}_{b}\right) \chi_{b}\left(\boldsymbol{r}_{b}, \boldsymbol{r}_{b}^{\prime}, i \omega\right) v\left(\boldsymbol{r}_{b}^{\prime}, \boldsymbol{r}_{a}\right),
\end{aligned}
$$

where $\chi_{a}$ and $\chi_{b}$ are the linear density response functions of fragments $\mathcal{S}_{a}$ and $\mathcal{S}_{b}$, respectively, and $v\left(\boldsymbol{r}, \boldsymbol{r}^{\prime}\right)=\left|\boldsymbol{r}-\boldsymbol{r}^{\prime}\right|^{-1}$ is the bare Coulomb interaction. Position vectors $\boldsymbol{r}_{a}, \boldsymbol{r}_{a}^{\prime}$ are restricted to fragment $\mathcal{S}_{a}$ while $\boldsymbol{r}_{b}, \boldsymbol{r}_{b}^{\prime}$ are restricted to fragment $\mathcal{S}_{b}$. Equation (1) corresponds to the ZarembaKohn [26] (ZK) formula which was derived following secondorder perturbation theory. The ZK expression corresponds to the dispersion energy between two neutral polarizable fragments in terms of the charge fluctuations of each fragment. Within the adiabatic-connection fluctuation-dissipation theorem (ACFDT) formalism, Dobson [47] showed that the ZK formula is obtained when the response function is formulated in terms of the random-phase approximation (RPA).

We consider now the response function of each fragment $\mathcal{S}_{i}$ to be characterized by an isotropic point dipole polarizability $\alpha^{i}(i \omega)$ located at $\boldsymbol{R}_{i}[47]$ :

$$
\chi_{i}\left(\boldsymbol{r}_{i}, \boldsymbol{r}_{i}^{\prime}, i \omega\right)=-\alpha^{i}(i \omega) \nabla_{\boldsymbol{r}_{i}} \delta^{3}\left(\boldsymbol{r}_{i}-\boldsymbol{R}_{i}\right) \otimes \nabla_{\boldsymbol{r}_{i}^{\prime}} \delta^{3}\left(\boldsymbol{r}_{i}^{\prime}-\boldsymbol{R}_{i}\right),
$$

where $\delta^{3}\left(\boldsymbol{r}-\boldsymbol{r}^{\prime}\right)$ is the three-dimensional Dirac delta function and $\otimes$ corresponds to the tensor (outer) product. Given Eq. (2) for $\chi_{a}$ and $\chi_{b}$, the vdW interaction for two well-separated fragments given by (1) becomes [47]

$$
E_{\mathrm{vdW}}^{(2)} \simeq-\frac{3}{\pi R^{6}} \int_{0}^{\infty} d \omega \alpha^{a}(i \omega) \alpha^{b}(i \omega)=-\frac{C_{6}^{a b}}{R^{6}},
$$

where the Casimir-Polder formula [48] has been used to calculate $C_{6}^{a b}$ from the dipole polarizabilities of each fragment and $R=\left|\boldsymbol{R}_{a}-\boldsymbol{R}_{b}\right|$. The equation above corresponds to the pairwise formula known since the work of London [49] and, as it has been summarized by Dobson [50], can be derived in several ways. For the general case of $N$ polarizable dipoles in the well-separated regime, Tkatchenko and co-workers [51] showed that the second-order expansion of the correlation energy given within the ACFDT-RPA scheme leads to [51,52]

$$
E_{\mathrm{vdW}}^{(2)}=E_{c, \mathrm{RPA}}^{(2)}=-\frac{1}{2} \sum_{i} \sum_{j} \frac{C_{6}^{i j}}{R_{i j}^{6}} .
$$

The reader will note that Eq. (4) corresponds to the expression for the pairwise dispersion energy of $N$ atoms as used in the DFT-D [12,53,54] and DFT+vdW [17] methods.

\section{B. Atom-surface van der Waals interaction}

The vdW interaction between a semi-infinite crystalline solid and a neutral atom can be derived starting from Eq. (1) in the limit where there is no wave-function overlap between the atom and the surface [26]. Figure 1 features the geometric arrangement of the atom-surface system. In this arrangement, fragment $\mathcal{S}_{a}$ is the atom located at a distance $Z$ from the topmost layer of the surface, which corresponds to fragment $\mathcal{S}_{S}$. The origin of the coordinate system is chosen to lie in the plane of the topmost surface atoms. For the case of the atom-surface interaction, the $\mathrm{ZK}$ formula correlates the charge 


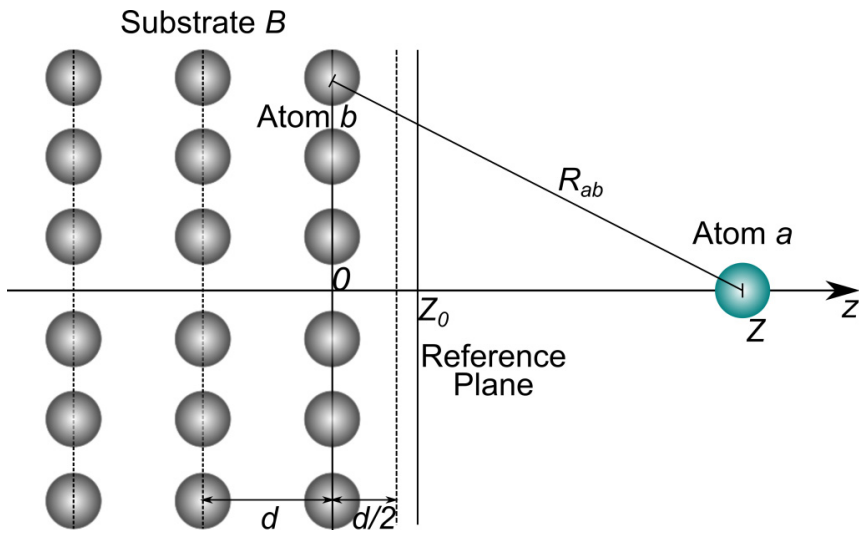

FIG. 1. Geometry of the atom-surface system.

fluctuations between adsorbate and substrate and does not rely on any specific model for any of the involved fragments [40]. In this case, the Coulomb potential between fragments can be expressed in terms of two-dimensional Fourier decompositions in order to incorporate the symmetry of the planar semi-infinite substrate. After expressing the Coulomb potential in terms of the two-dimensional (2D) wave vector $\boldsymbol{q}$, which lies parallel to the plane of the surface, and incorporating the complex wave vector $\boldsymbol{\kappa}=\boldsymbol{q}+i q \hat{\boldsymbol{z}}$, the atom-surface dispersion interaction takes the form $[26,40]$

$$
\begin{aligned}
E_{\mathrm{vdW}}^{(2)}= & -\frac{1}{2 \pi} \int_{0}^{\infty} d \omega \int \frac{d^{2} q}{(2 \pi)^{2}} \int \frac{d^{2} q^{\prime}}{(2 \pi)^{2}} \frac{2 \pi}{q} \frac{2 \pi}{q^{\prime}} e^{-Z\left(q+q^{\prime}\right)} \\
& \times e^{i \boldsymbol{R} \cdot\left(\boldsymbol{q}-\boldsymbol{q}^{\prime}\right)} A\left(\boldsymbol{x}, \boldsymbol{x}^{\prime}, i \omega\right) S\left(\boldsymbol{r}, \boldsymbol{r}^{\prime}, i \omega\right)
\end{aligned}
$$

$A\left(\boldsymbol{x}, \boldsymbol{x}^{\prime}, i \omega\right)$ represents the adsorbate response function and is given by

$$
A\left(\boldsymbol{x}, \boldsymbol{x}^{\prime}, i \omega\right)=\int d \boldsymbol{x} \int d \boldsymbol{x}^{\prime} e^{i \kappa \cdot \boldsymbol{x}-i \kappa^{*} \cdot \boldsymbol{x}^{\prime}} \chi_{a}\left(\boldsymbol{x}, \boldsymbol{x}^{\prime}, i \omega\right),
$$

where the position vector $\boldsymbol{x}$ is taken relative to the center of the adatom located at $\boldsymbol{R}=(0,0, Z)$, that is $\boldsymbol{x}=\boldsymbol{r}-\boldsymbol{R}$. The surface response function $S\left(\boldsymbol{r}, \boldsymbol{r}^{\prime}, i \omega\right)$ is the analog to Eq. (6) and is given by

$$
S\left(\boldsymbol{r}, \boldsymbol{r}^{\prime}, i \omega\right)=\int d \boldsymbol{r} \int d \boldsymbol{r}^{\prime} e^{i \kappa \cdot \boldsymbol{r}-i \kappa^{*} \cdot \boldsymbol{r}^{\prime}} \chi_{S}\left(\boldsymbol{r}, \boldsymbol{r}^{\prime}, i \omega\right),
$$

where $\boldsymbol{r}=(\boldsymbol{\rho}, z)$. Due to the periodicity of the surface, the integral over $\boldsymbol{q}^{\prime}$ in Eq. (5) is restricted to $\boldsymbol{q}^{\prime}=\boldsymbol{q}+\boldsymbol{G}$, where $\boldsymbol{G}$ is a reciprocal lattice vector in the plane of the surface. Only the terms where $\boldsymbol{G}=0$ give rise to a power-law dependence characteristic of the vdW interaction [26]. Taking only the $\boldsymbol{G}=0$ terms, Eq. (5) reduces to [40]

$$
E_{\mathrm{vdW}}^{(2)}=-\frac{1}{2 \pi} \int_{0}^{\infty} d \omega \int \frac{d^{2} q}{(2 \pi)^{2}} \frac{2 \pi}{q} e^{-2 q Z} A(q, i \omega) S(q, i \omega) .
$$

The factor $e^{-2 q Z}$ appearing in Eq. (8) cuts off the sum of $q$ values to $q \gtrsim 1 / Z$. Therefore, it is sufficient to determine only the small- $q$ behavior of functions $A(q, i \omega)$ and $S(q, i \omega)$. $A(q, i \omega)$ contains the fluctuations in the density of the adsorbate due to the dipole and higher-multipole moments of the atom. It can be expanded in terms of even powers of $q$ as $[26,40]$

$$
A(q, i \omega)=2 \alpha_{1}^{a}(i \omega) q^{2}+\frac{2}{3} \alpha_{2}^{a}(i \omega) q^{4}+\mathcal{O}\left(q^{6}\right)+\ldots,
$$

where $\alpha_{1}^{a}$ and $\alpha_{2}^{a}$ are the frequency-dependent dipole and quadrupole polarizabilities of atom $a$, respectively, evaluated at imaginary frequency. Higher-multipole polarizabilities correspond to higher even powers of $q$.

The surface response function $S(q, i \omega)$, which is given by

$$
S(q, i \omega)=\frac{2 \pi}{q} \int d z \int d z^{\prime} e^{q\left(z+z^{\prime}\right)} \chi_{S}\left(z, z^{\prime}, i \omega\right),
$$

contains all the information of the substrate, that is, its structure and the response given by its electronic structure. $S(q, i \omega)$ can also be formulated as [26,55]

$$
S(q, i \omega)=\int d z e^{q z} \delta n(z, q, i \omega),
$$

where $\delta n(z, q, i \omega)$ is the surface electron density induced by an external charge of the form $[26,55] \rho_{\text {ext }}(\boldsymbol{r}, t)=\delta(z-$ $Z) e^{i \boldsymbol{q} \cdot \rho} e^{\omega t}$. The surface response function gives the relative amplitude of the induced electrostatic potential. It can also be seen, according to Eq. (11), as an exponentially weighted integral of the surface charge density [55]. It includes effects due to the diffuseness of the surface charge density and due to the nonlocal dielectric response of the surface and the bulk. Equation (11) can be expanded in terms of $q$ as [40]

$$
S(q, i \omega)=\sigma_{0}(i \omega)+q \sigma_{1}(i \omega)+\mathcal{O}\left(q^{2}\right)+\ldots,
$$

where the term $\sigma_{0}$, corresponding to the $q=0$ limit of $S(q, i \omega)$, is the total surface charge density and can be expressed in terms of the bulk dielectric function $\varepsilon_{S}(i \omega)$ of substrate $S$ as

$$
\sigma_{0}(i \omega)=\frac{\varepsilon_{S}(i \omega)-1}{\varepsilon_{S}(i \omega)+1} .
$$

The expansion of $S(q, i \omega)$ in powers of $q$ generates a series for $E_{\mathrm{vdW}}^{(2)}$ which can be in consequence given in terms of inverse powers of $Z$ [26,40]. Given the expansions of Eqs. (9) and (12), the vdW interaction of Eq. (8) can be expressed as

$$
E_{\mathrm{vdW}}^{(2)} \simeq-\frac{C_{3}^{a S}}{Z^{3}}-\frac{C_{4}^{a S}}{Z^{4}}-\mathcal{O}\left(Z^{-5}\right)+\ldots,
$$

where

$$
C_{3}^{a S}=\frac{1}{4 \pi} \int_{0}^{\infty} d \omega \alpha_{1}^{a}(i \omega) \sigma_{0}(i \omega)
$$

and

$$
C_{4}^{a S}=\frac{3}{8 \pi} \int_{0}^{\infty} d \omega \alpha_{1}^{a}(i \omega) \sigma_{1}(i \omega) .
$$

The leading term of Eq. (14) shows the characteristic $Z^{-3}$ behavior of the atom-surface vdW interaction [25,26,56], which depends on the dipole polarizability of the adsorbate and the surface charge density of the substrate [Eq. (15)]. Higher$Z^{-n}$ terms and their respective interaction coefficients $C_{n}^{a S}$ correspond to complex expressions involving both adsorbate and substrate response properties [40].

With the identification of $\sigma_{0}$ in terms of the macroscopic bulk dielectric function of the substrate in Eq. (13), the 
interaction coefficient $C_{3}^{a S}$ is given by

$$
C_{3}^{a S}=\frac{1}{4 \pi} \int_{0}^{\infty} d \omega \alpha_{1}^{a}(i \omega) \frac{\varepsilon_{S}(i \omega)-1}{\varepsilon_{S}(i \omega)+1} .
$$

Lifshitz [25] originally gave a macroscopic formulation of the attractive vdW forces between two bodies. His formulation characterized each body in terms of spatially nondispersive ( $q=0$ ) frequency-dependent dielectric functions such as $\sigma_{0}$ in Eq. (17) and resulted in the same inverse third power dependence on the distance between bodies. The approach of Zaremba and Kohn that yields the asymptotic expansion in Eq. (14) has the advantage of taking into account the microscopic details of the surface in the atom-surface vdW interaction [26]. However, Eq. (14) cannot be applied directly to the atom-surface vdW interaction since the choice of the origin of coordinates in the expansion is not obvious considering the distances typically found in physisorption. The first two terms in Eq. (14) can be recovered by writing [26,40]

$$
E_{\mathrm{vdW}}^{(2)} \simeq-\frac{C_{3}^{a S}}{\left(Z-Z_{0}\right)^{3}},
$$

where $Z_{0}$ is the position of the reference plane for the atomsurface vdW interaction and is defined as

$$
Z_{0} \equiv \frac{C_{4}^{a S}}{3 C_{3}^{a S}}
$$

The vdW reference plane $Z_{0}$ can be understood as a consequence of the spatially dispersive character of the substrate's density response function as its definition in terms of $C_{4}^{a S}$ indicates $[26,40]$. The physical importance of $C_{4}^{a S}$ lies in its dependence on $\sigma_{1}$ in Eq. (16), which corresponds to the linear term in $q$ found in the expansion of the surface response function $S(q, i \omega)$. We note that the relationship among the Lifshitz theory, the ZK theory, and the RPA approximation within the ACFDT formalism is discussed by Dobson and Gould in a recent paper [57].

\section{Atom-surface vdW interaction as a sum of interatomic pairwise potentials}

In order to give a theoretical basis to Polanyi's potential theory of adsorption [58,59], London and Polanyi [56] first proposed the inverse third power dependence on the distance for the adsorption of particles in a gas on a flat surface. Based on the work in dispersion forces between atoms by London [49], their approach consisted in the summation of pairwise vdW interactions between a single gas particle and each of the atoms contained in the volume of the solid, yielding an inverse third power dependence on the distance between particle and substrate.

Let us consider a homogeneous distribution of attractive forces within the substrate between atom $a$ and each of the atoms $s$ constituting substrate $S$ given by the leading $-C_{6} R^{-6}$ term of the vdW interaction between two atoms. We can recover the inverse third power dependence on the distance by integrating the pairwise interaction over the volume of the substrate spanning the region $\mathcal{S}_{S}[60,61]$ :

$$
E_{\mathrm{vdW}}^{(2)} \simeq-\int_{\mathcal{S}_{S}} d V n_{S} \frac{C_{6}^{a s}}{R^{6}},
$$

where $d V$ is the volume element of substrate $S$ and $n_{S}$ is the number of atoms per unit volume in the bulk of the substrate. Starting from Eq. (3), the LZK formula given in Eq. (18) can be recovered exactly $[26,40,62]$ by setting (i)

$$
C_{3}^{a S}=n_{S}\left(\frac{\pi}{6}\right) C_{6}^{a s},
$$

and (ii) $Z_{0}=d / 2$, where $d$ is the interlayer distance between equally spaced lattice planes parallel to the surface. In the jellium model of a metal, $d / 2$ corresponds to the position of the jellium edge $[26,40]$. Deviations from this position occur due to local field effects in the dielectric function and reflect surface polarization. The magnitude of these deviations also constitutes a measure of the importance of many-body forces in the potential between the atom and the solid [26].

\section{DFT $+v_{d W} \mathbf{W}^{\text {surf }}$ method for modeling adsorption phenomena}

Our discussion of vdW interactions so far has emphasized the well-separated regime where there is no wave-function overlap between the interacting fragments. This regime is a natural starting point for $\mathrm{vdW}$ interactions as the origin of these lies on the induced polarization which results from instantaneous fluctuations of the electronic density, effects which are part of the electronic correlation energy of the interacting system. However, in order to provide a quantitative account even in model adsorption systems, such as the adsorption of noble gases on metal surfaces, it is necessary to incorporate the (chemical) interactions that occur at the short-range regime where the wave-function hybridization becomes important. This can be understood in the context of electronic-structure theory as treating the exchange and correlation energies on an equal footing.

It is evident that the main challenge in the modeling of the adsorption of atoms and molecules on surfaces is to develop methods that are able to capture both covalent and noncovalent interactions in a reliable manner while at the same time being capable of dealing with realistic adsorption systems in an efficient fashion. It is with these features in mind that we present in this section a method to model screened $\mathrm{vdW}$ interactions for the adsorption of atoms and molecules on surfaces: the DFT+vdW ${ }^{\text {surf }}$ method. The DFT $+v \mathrm{dW}^{\text {surf }}$ scheme combines methods for molecules and solids with the purpose of an accurate description of $\mathrm{vdW}$ interactions in modeling the adsorption of atoms and molecules on surfaces. We accomplish this by linking the LZK theory of the vdW interaction between an atom and a solid surface to include the collective response of the substrate electrons with the TS DFT + vdW method to include intermolecular interactions.

The DFT $+\mathrm{vdW}^{\text {surf }}$ method consists in a vdW energy correction to the total DFT energy, where the vdW energy of the system is calculated as a sum of pairwise interaction terms:

$$
E_{\mathrm{vdW}}=-\frac{1}{2} \sum_{a} \sum_{b} f_{\mathrm{damp}}\left(R_{a b}, R_{a}^{0}, R_{b}^{0}\right) \frac{C_{6}^{a b}}{R_{a b}^{6}},
$$

where $R_{a b}$ is the distance between atoms $a$ and $b$ and $C_{6}^{a b}$ is the corresponding $C_{6}$ coefficient given by the Casimir-Polder integral of Eq. (3). The damping function $f_{\text {damp }}$ eliminates 
the $R_{a b}^{-6}$ singularity found at small distances and is a function of the vdW radii $R_{a}^{0}$ and $R_{b}^{0}$. In analogy to the DFT+vdW method, we adopt a Padé approximant model [63] for the frequency-dependent dipole polarizability of atom $i=\{a, b\}$ given by the leading term of the Padé series as

$$
\alpha_{1}^{i}(\omega)=\frac{\alpha_{0}^{i}}{1-\left(\omega / \eta_{i}\right)^{2}},
$$

where $\alpha_{0}^{i}$ is the static dipole polarizability of atom $i$ and $\eta_{i}$ is an effective excitation frequency $[17,64]$. The Casimir-Polder integral can be solved analytically with $\alpha_{1}(i \omega)$ given by Eq. (23) leading to a London-type formula [17,64]

$$
C_{6}^{a b}=\frac{3}{2}\left(\frac{\eta_{a} \eta_{b}}{\eta_{a}+\eta_{b}}\right) \alpha_{0}^{a} \alpha_{0}^{b},
$$

with the effective frequency of atom $i$ given when $a=b$ as [17]

$$
\eta_{i}=\frac{4}{3} \frac{C_{6}^{i i}}{\left(\alpha_{0}^{i}\right)^{2}} .
$$

With Eqs. (24) and (25), a simple combination rule for $C_{6}^{a b}$ is found [17]:

$$
C_{6}^{a b}=\frac{2 C_{6}^{a a} C_{6}^{b b}}{\frac{\alpha_{0}^{b}}{\alpha_{0}^{a}} C_{6}^{a a}+\frac{\alpha_{0}^{a}}{\alpha_{0}^{b}} C_{6}^{b b}} .
$$

Equation (26) gives the interaction coefficient $C_{6}^{a b}$ between atoms $a$ and $b$ in terms of the homonuclear parameters $C_{6}^{i i}$ and $\alpha_{0}^{i}$. We will refer to these in the following as vdW parameters.

\section{Reference $v d W$ parameters}

For the case of free-atom reference vdW parameters, accurate values are given in the database of Chu and Dalgarno [65] (see also Ref. [17]). In the case of solids, the reference vdW parameters for an atom must be determined taking into account the environmental effects that an atom-in-a-solid is subject to [66]. We rely on the LZK theory to achieve this. We start by noting that for the atom-surface vdW interaction, we can recover the LZK formula (18) exactly starting from a summation of pairwise potentials between the adsorbate and each of the atoms in the solid. The pairwise $C_{6}^{a s}$ coefficient between atom $a$ and atom $s$ in the solid can be obtained from Eqs. (21) and (17) as

$$
\begin{aligned}
C_{6}^{a s} & =\frac{1}{n_{S}}\left(\frac{6}{\pi}\right) C_{3}^{a S} \\
& =\frac{1}{n_{S}}\left(\frac{3}{2 \pi^{2}}\right) \int_{0}^{\infty} d \omega \alpha_{1}^{a}(i \omega) \frac{\varepsilon_{S}(i \omega)-1}{\varepsilon_{S}(i \omega)+1} .
\end{aligned}
$$

The effective vdW coefficient $C_{6}^{a s}$ given in Eq. (27) inherits the many-body collective response (screening) of the solid as indicated by its dependence on the dielectric function $\varepsilon_{S}$. In this context, the adsorbate corresponds to a free atom in the gas phase, which allows us to evaluate $\alpha_{1}^{a}(i \omega)$ with Eq. (23) using the values of $C_{6}^{a a}$ and $\alpha_{0}^{a}$ given by Chu and Dalgarno [65]. Equation (27) can then be determined by calculating the dielectric function $\varepsilon_{S}(i \omega)$ of the solid. We use the KramersKronig relation to determine $\varepsilon_{S}(i \omega)$ in terms of the absorptive part of the dielectric function $\varepsilon_{2}$ at real frequencies. We mainly take data from reflection energy-loss spectroscopy (REELS)
TABLE I. Screened vdW parameters as used in the DFT+vdW method. For comparison, the free-atom parameters as used in the DFT+vdW method are also shown. $C_{6}$ (in hartree bohr ${ }^{6}$ ), $\alpha_{0}$ (in bohr $^{3}$ ), and $R^{0}$ (in bohr) denote the dispersion coefficient, polarizability, and vdW radius, respectively. The experimental lattice constants [70] have been employed to calculate $n_{S}$ in Eq. (21).

\begin{tabular}{lccccccc}
\hline \hline & \multicolumn{3}{c}{ Screened } & & \multicolumn{3}{c}{ Free atom } \\
\cline { 2 - 4 } \cline { 6 - 7 } Substrate & $C_{6}$ & $\alpha_{0}$ & $R^{0}$ & & $C_{6}$ & $\alpha_{0}$ & $R^{0}$ \\
\hline $\mathrm{Ti}$ & 116 & 16.8 & 2.51 & & 1044 & 98.0 & 4.51 \\
$\mathrm{~V}$ & 80 & 13.3 & 2.40 & & 832 & 84.0 & 4.44 \\
$\mathrm{Fe}$ & 61 & 11.0 & 2.46 & & 482 & 56.0 & 4.23 \\
$\mathrm{Co}$ & 55 & 10.5 & 2.50 & & 408 & 50.0 & 4.18 \\
$\mathrm{Ni}$ & 59 & 10.2 & 2.28 & & 373 & 48.0 & 3.82 \\
$\mathrm{Cu}$ & 59 & 10.9 & 2.40 & & 253 & 42.0 & 3.76 \\
$\mathrm{Zn}$ & 62 & 12.9 & 2.76 & & 284 & 40.0 & 4.02 \\
$\mathrm{Ru}$ & 53 & 13.6 & 2.36 & & 610 & 65.9 & 4.00 \\
$\mathrm{Rh}$ & 84 & 13.0 & 2.42 & & 469 & 56.1 & 3.95 \\
$\mathrm{Pd}$ & 102 & 13.9 & 3.07 & & 158 & 23.7 & 3.66 \\
$\mathrm{Ag}$ & 122 & 15.4 & 2.57 & & 339 & 50.6 & 3.82 \\
$\mathrm{Ir}$ & 98 & 13.2 & 2.71 & & 359 & 42.5 & 4.00 \\
$\mathrm{Pt}$ & 120 & 14.5 & 2.80 & & 347 & 39.7 & 3.92 \\
$\mathrm{Au}$ & 134 & 15.6 & 2.91 & & 298 & 36.5 & 3.86 \\
\hline \hline
\end{tabular}

experiments by Werner and co-authors [67] for this purpose. In the case of $\mathrm{Rh}$ and $\mathrm{Ir}$, optical constants were taken from the reflectance measurements of Windt and co-authors [68]. Finally, in the case of $\mathrm{Ru}$, the optical measurements were taken from Choi and co-authors [69]. We may note in passing that the determination of the dielectric function as input for the coefficients in the present method is not limited to experimental results. It may also be accurately computed from first principles as demonstrated by Werner and co-workers [67], whose DFT calculations agree reasonably well with REELS results within the experimental uncertainties involved.

Having determined $C_{6}^{a s}$, the reference vdW parameters $C_{6}^{s s}$ and $\alpha_{0}^{s}$ for the atom-in-a-solid can be calculated by a system of two equations like Eq. (26) with $b=s$ and two different adsorbing atoms $a$. Take, for example, $\mathrm{Cu}$ interacting with $\mathrm{Ne}$ and Ar. Two equations of the type given by Eq. (26) with $s=$ $\mathrm{Cu}$ can be set for $C_{6}^{\mathrm{NeCu}}$ and $C_{6}^{\mathrm{ArCu}}$ where $C_{6}^{\mathrm{CuCu}}$ and $\alpha_{0}^{\mathrm{Cu}}$ are the only two unknown parameters. We take any two atoms from the list: $\mathrm{H}, \mathrm{C}, \mathrm{Ne}, \mathrm{Ar}$, and $\mathrm{Kr}$ and solve the set of two equations for $C_{6}^{s s}$ and $\alpha_{0}^{s}$ for a given substrate. The resulting vdW reference parameters of different substrate atoms are displayed in Table I. For comparison, the reference parameters of the free atoms are presented as well. The vdW radius for the atom-in-a-solid $R_{s}^{0}$ is obtained via the relation $R_{s}^{0}=\left(\alpha_{0}^{s} / \alpha_{0 \text {,free }}^{s}\right)^{1 / 3} R_{s \text {,free }}^{0}$ where $R_{s, \text { free }}^{0}$ corresponds to the vdW radius of the same species $s$ but as a free atom. We use the TS ansatz [17] to determine the free-atom vdW radii.

The values in Table I for the screened vdW parameters for an atom-in-a-solid show that the environmental effects in a solid cannot be neglected in the calculation of $\mathrm{vdW}$ interactions. The inclusion of the collective response of the solid in the determination of the $\mathrm{vdW}$ parameters for transition metals can lead to pronounced differences with respect to the free-atom reference values, reducing the vdW $C_{6}$ coefficients 
up to a factor of 10. Significant effects can be observed in static polarizabilities $\left(\alpha_{0}\right)$ and $\mathrm{vdW}$ radii $\left(R^{0}\right)$ as well. The sensitive dependence of the dielectric screening on the substrate is manifested clearly by these results. We note that the parameters here calculated can be considered as intrinsic properties of the bulk as they are essentially invariant to the nature of the adsorbed atom.

\section{Hybridization and interface polarization effects}

A set of accurate reference vdW parameters has been established so far for both free atoms and atoms inside a solid. However, the effects of charge polarization that an atom in a molecule or an interface would experience are not included yet. In the case of adsorption phenomena, there will be effects related to the polarization of the interface. These effects are manifested as the spatial dispersion in the dielectric function close to the surface of the system. They are included in higher $q$-dependent terms of the substrate response function given by Eq. (12).

The effects of charge polarization are included in the case of molecules in the DFT+ vdW method [17] by renormalizing the vdW parameters using the ground-state electron density obtained from DFT calculations. We adopt the same strategy to account for interface polarization in adsorption phenomena by defining an effective volume $v_{\mathrm{eff}}^{i}$ for species $i$ as

$$
\begin{gathered}
v_{\mathrm{eff}}^{i}=\frac{V_{i}^{\mathrm{eff}}}{V_{i}^{\mathrm{ref}}}=\left(\frac{\int d \boldsymbol{r} r^{3} w_{i}(\boldsymbol{r}) n(\boldsymbol{r})}{\int d \boldsymbol{r} r^{3} n_{i}^{\mathrm{ref}}(\boldsymbol{r})}\right), \\
w_{i}(\boldsymbol{r})=\frac{n_{i}^{\mathrm{ref}}(\boldsymbol{r})}{\sum_{j} n_{j}^{\mathrm{ref}}(\boldsymbol{r})},
\end{gathered}
$$

where $r^{3}$ is the cube of the distance from the nucleus of atom $i, w_{i}(\mathbf{r})$ is the Hirshfeld [71] atomic partitioning weight of the species $i, n(\mathbf{r})$ is the total electron density, $n_{i}^{\text {ref }}(\mathbf{r})$ is the reference electron density for atom $i$, and the sum goes over all atoms of the system $[17,72]$. For the solid, the reference corresponds to the spherical electron density of an atom in the bulk, and for a molecule, it corresponds to the free-atom electron density. By exploiting the direct relation between polarizability and volume $[17,73]$, the effective $C_{6 \text {,eff }}$ coefficient, the effective polarizability $\alpha_{\text {eff }}$, and the effective $\mathrm{vdW}$ radius $R_{i, \mathrm{eff}}^{0}$ are determined as [17]

$$
\begin{gathered}
C_{6, \text { eff }}^{i i}=\left(v_{\text {eff }}^{i}\right)^{2} C_{6, \text { ref }}^{i i}, \\
\alpha_{\text {eff }}^{i}=\left(v_{\text {eff }}^{i}\right) \alpha_{\text {ref }}^{i}, \\
R_{i, \text { eff }}^{0}=\left(\frac{\alpha_{\text {eff }}^{i}}{\alpha_{\text {ref }}^{i}}\right)^{1 / 3} R_{i, \text { ref }}^{0} .
\end{gathered}
$$

Effects beyond the pairwise approximation are achieved by the inclusion of semilocal effects through the dependence of the vdW parameters on the electron density as given by Eqs. (30)-(32). For example, we have reported significant interface polarization in systems such as PTCDA on $\mathrm{Ag}(111)$ and benzene on $\operatorname{Pt}(111)$ manifested in the value of the $C_{6}$ coefficients in the region of the metal-molecule interface [22,29].

\section{Empirical short-range damping function}

The fact that we adopt an interatomic pairwise expression as Eq. (22) in order to compute vdW interactions leads to the presence of a $R_{a b}^{-6}$ singularity at small distances. We also couple to a semilocal xc functional via the short-range damping function. The damping function $f_{\text {damp }}$ in the $\mathrm{DFT}+\mathrm{vdW} \mathrm{W}^{\text {surf }}$ method follows the same strategy as the DFT+vdW method, having the following form [17];

$$
f_{\text {damp }}\left(R_{a b}, R_{a b}^{0}\right)=\frac{1}{1+\exp \left[-d\left(\frac{R_{a b}}{s_{R} R_{a b}^{0}}-1\right)\right]},
$$

where $R_{a b}^{0}=R_{a}^{0}+R_{b}^{0}, d=20$ determines the steepness of the damping, and $s_{R}$ determines the range of the damping. The range parameter $s_{R}$ is the only parameter that is determined empirically. This is done by fitting $s_{R}$ for each underlying xc functional to the S22 data set of Jurečka and co-authors [74]. The S22 data set contains binding energies of 22 different weakly bound systems, calculated using the coupled-cluster method with single, double, and triple excitations, where triple excitations are treated perturbatively $[\mathrm{CCSD}(\mathrm{T})]$ (see Refs. [17,75]).

The DFT $+\mathrm{vdW}^{\text {surf }}$ method leads to a reduced set of effective $C_{6}$ coefficients (see Table I) that are determined by the dielectric screening of the bulk and the electronic environment of each atom, yielding a smaller long-range vdW energy. On the other hand, it also carries a larger relative weight of the vdW contributions at shorter range due to the effect that the reduction of the vdW radii has on the damping function. The nontrivial coaction of these effects and the underlying xc functional enables an accurate treatment of complex interfaces where the interplay of different interactions is present. We also mention that the DFT $+\mathrm{vdW}^{\text {surf }}$ method does not depend on the nature of the substrate, and is in principle equally applicable to insulators, semiconductors, and metals [22].

\section{Metal bulk lattice constants with the DFT $+v^{\text {surf }}$ method}

We have calculated the bulk lattice constant of several transition metals in a previous work [29] using the $\mathrm{DFT}+\mathrm{vdW}^{\text {surf }}$ method with the PBE approximation as underlying $\mathrm{xc}$ functional, which we refer to as $\mathrm{PBE}+\mathrm{vdW} \mathrm{Wurf}^{\text {sur }}$. As we have discussed above, the $P B E+v d W^{\text {surf }}$ method includes the screening due to metallic bulk electrons in the computation of the long-range vdW energy tail. However, since the PBE functional is reduced to the local-density approximation (LDA) for homogeneous electron densities, the metallic electrons are already accurately described within the PBE functional. This fact results in a partial "double counting" of the interaction between metallic electrons with the $\mathrm{PBE}+\mathrm{vdW}$ surf method, leading to an overestimation of the vdW energy inside the metal bulk. This effect yields a slight increase of the lattice constants compared to the PBE functional in some transition metals while decreasing it in some other cases (see Ref. [29]). But, even if the present method can actually lead to an improvement in the bulk lattice constant for some of the transition metals here studied, there is no straightforward way to quantify the overestimation effects. On the other hand, these effects do not pose a problem in the adsorption of molecules on surfaces 
because the adsorbate interacts both with the localized ions and the delocalized metallic electrons. Further improvement of the lattice constants requires a full microscopic treatment of the polarizability due to localized ions and metallic electrons.

\section{RESULTS AND DISCUSSION}

We have developed in Sec. II a method within DFT that includes screened vdW interactions for treating adsorption phenomena. In this section, we apply this methodology and assess its performance in realistic adsorption systems. Before addressing the case of HIOS, the effects of modeling $\mathrm{vdW}$ interactions within DFT in adsorption phenomena can be evaluated by investigating the interaction between noble gases and a metallic surface. The adsorption interaction in these prototypical physisorption systems is the result of a balance between attractive vdW interactions and Pauli repulsion. We start this section by describing the specifications of the systems studied here and the computational details of our calculations. We continue by analyzing the adsorption of $\mathrm{Xe}$ on selected transition-metal surfaces using the $\mathrm{PBE}+\mathrm{vdW}$ and PBE $+v^{2} W^{\text {surf }}$ methods, discussing the differences between the two methods while taking into consideration that the latter includes the collective response of the substrate electrons in the determination of the vdW parameters. We have presented some of these results in Ref. [22], but here we extend the analysis by including PBE + vdW calculations and studying the perpendicular vibrational energy of $\mathrm{Xe}$ in each case to probe the curvature around the minimum of the potential-energy curves calculated with the $\mathrm{PBE}+\mathrm{vdW}$ surf method. As a next step, it is illustrative to study a typical physisorbed organic/metal interface for which we have chosen the adsorption of a single molecule of PTCDA on Au(111). We proceed by addressing the performance of the $\mathrm{PBE}+\mathrm{vdW}$ surf method for adsorption on non-close-packed surfaces. For this, we first analyze the differences between the adsorption of Xe on $\mathrm{Cu}(111)$ and $\mathrm{Cu}(110)$. As a second case, we analyze the adsorption of an organic adsorbate on a metallic surface with different orientations. We take the interface formed by the adsorption of a PTCDA monolayer on the $\mathrm{Ag}(111), \operatorname{Ag}(100)$, and $\operatorname{Ag}(110)$ surfaces.

\section{A. System specifications and calculation details}

The DFT calculations were performed using the allelectron/full-potential electronic-structure code FHI-AIMS [76] which uses efficient numerical atom-centered orbitals (NAO) as basis set. We used the tight settings in the FHI-AIMS code for all calculations. These include the tier 1 standard basis set for the transition metals and $\mathrm{Xe}$, and the tier 2 basis set for $\mathrm{C}, \mathrm{H}$, and $\mathrm{O}$. The convergence criteria in the calculations were $10^{-5}$ electrons for the electron density and $10^{-6} \mathrm{eV}$ for the total energy of the system. For all structure relaxations, $0.01 \mathrm{eV} \AA^{-1}$ was utilized as convergence criterion for the maximum final force. Relativistic effects were included via the atomic scalar zeroth-order regular approximation [77]. We used the repeated-slab method to model all the systems together with the PBE [78] exchange-correlation functional.

The vdW ${ }^{\text {surf }}$ method includes the screening due to metallic bulk electrons in the computation of the long-range vdW energy tail. However, since the PBE functional is reduced to the local-density approximation (LDA) for homogeneous electron densities, the metallic electrons are already accurately described within the PBE functional. This fact results in a partial "double counting" of the interaction between metallic electrons with the $\mathrm{PBE}+\mathrm{vdW}$ surf method, leading to an overestimation of the vdW energy inside the metal bulk [29]. Even if the present method can lead to an improvement in the bulk lattice constant for some of the transition metals here studied [29], there is no straightforward way to quantify the overestimation effects. For this reason, we have used the PBE optimized lattice constant to generate most of the metal slabs [29]: 4.149, 3.631, 3.971, and $3.943 \AA$ for Ag, $\mathrm{Cu}, \mathrm{Pt}$, and $\mathrm{Pd}$, respectively. The only exception is $\mathrm{Au}$ in the study of PTCDA on Au(111). In this case, we used the experimental lattice constant of $\mathrm{Au}(4.065 \AA)$ to generate the (111) surface in order to have a direct comparison with the binding curve generated in the experiments performed by Wagner and co-workers [79].

For the case of $\mathrm{Xe}$ on metallic surfaces, we adopted the experimentally reported $(\sqrt{ } 3 \times \sqrt{ } 3) R 30^{\circ}$ structure with top and face-centered-cubic (fcc) hollow adsorption sites for the (111) surface of $\mathrm{Pt}, \mathrm{Pd}$, and $\mathrm{Cu}$. For the case of the $\mathrm{Cu}(110)$ surface, we present the results for a $2 \times 2$ surface unit cell. We used a Monkhorst-Pack grid [80] of $15 \times 15 \times 1 k$ points in the reciprocal space and six metallic layers to perform the calculations, except for $\mathrm{Cu}(110)$, where we used seven metallic layers. The width of the vacuum was $20 \AA$.

In the case of PTCDA on $\mathrm{Au}(111)$, the system consisted of a single molecule adsorbed on a $\mathrm{Au}(111)$ surface modeled with three metallic layers in line with previous investigations [19-22,81]. The surface unit cell was modeled with a $\left(\begin{array}{cc}6 & -6 \\ 1 & 10\end{array}\right)$ supercell and a vacuum width of $40 \AA$ in order to minimize the interactions between neighboring molecules. We used a Monkhorst-Pack grid of $2 \times 2 \times 1 k$ points in the reciprocal space for the DFT calculations.

Regarding PTCDA on silver surfaces, the surface unit cells were modeled with a $\left(\begin{array}{cc}6 & 1 \\ -3 & 5\end{array}\right),\left(\begin{array}{cc}4 & 4 \\ -4 & 4\end{array}\right)$, and a $\left(\begin{array}{cc}3 & 2 \\ -3 & 2\end{array}\right)$ supercell for the case of $\operatorname{Ag}(111), \operatorname{Ag}(100)$, and $\operatorname{Ag}(110)$, respectively, in accordance to experimental results $[82,83]$. The slabs consisted of five layers for $\operatorname{Ag}(111)$ and $\operatorname{Ag}(100)$ and seven layers for $\mathrm{Ag}(110)$, each with a vacuum gap of $50 \AA$. We used a Monkhorst-Pack grid of $4 \times 4 \times 1$ for $\mathrm{Ag}(111)$ and $6 \times 6 \times 1$ $k$ points for $\operatorname{Ag}(100)$ and $\operatorname{Ag}(110)$ in the reciprocal space.

\section{B. Xe on metal surfaces}

We performed $\mathrm{PBE}+\mathrm{vdW}$ and $\mathrm{PBE}+\mathrm{vdW}$ surf structure optimizations for Xe on five transition-metal surfaces, where the Xe atom and the atoms in the topmost and first subsurface layers of the metal slab were allowed to relax. As we generated the substrates using the PBE lattice constant, we did not consider vdW interactions between metal atoms in order to avoid an artificial relaxation of the surfaces. They were taken into account only in final adsorption energy calculations.

Adsorption energies. Figure 2 and Table II show the adsorption energies calculated with $\mathrm{PBE}+\mathrm{vdW} \mathrm{W}^{\text {surf }}$ for both the top and fcc hollow adsorption sites. The adsorption energies 


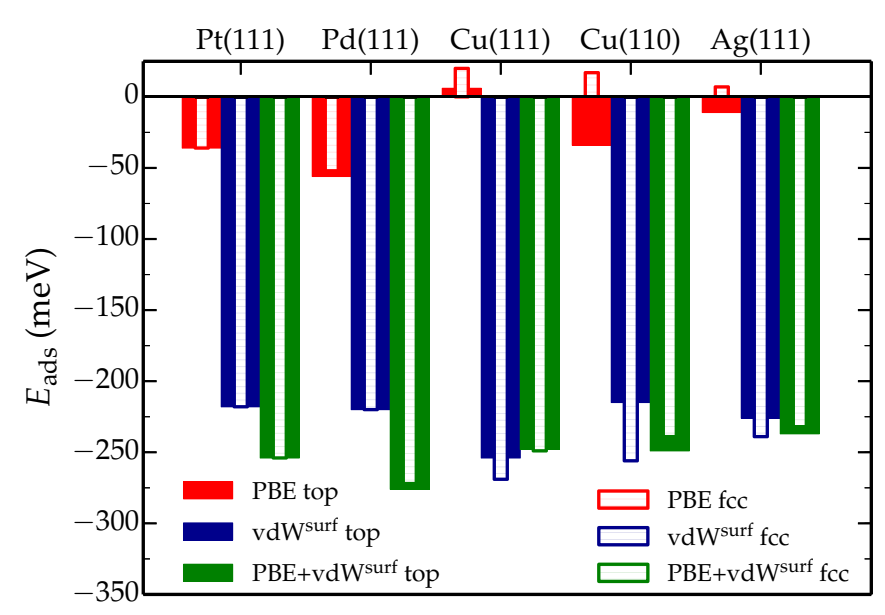

FIG. 2. Adsorption energies $E_{\text {ads }}$ calculated with $\mathrm{PBE}+\mathrm{vdW}$ surf for Xe on transition-metal surfaces. The contributions of PBE and $\mathrm{vdW}$ interactions after relaxing the system with the $\mathrm{PBE}+\mathrm{vdW} \mathrm{Wur}^{\text {sur }}$ method are shown in red and blue, respectively. Total adsorption energies after relaxation are displayed in green. Top sites are displayed with plain color filled bars whereas fcc hollow sites are displayed with pattern filled bars.

were computed using

$$
E_{\mathrm{ads}}=E_{\mathrm{AdSys}}-\left(E_{\mathrm{Me}}+E_{\mathrm{Ad}}\right),
$$

where $E_{\text {AdSys }}$ is the total energy of the adsorption system (gas + metal surface) after relaxation, $E_{\mathrm{Me}}$ is the energy of the bare slab after relaxation, and $E_{\mathrm{Ad}}$ is the energy of the isolated $\mathrm{Xe}$ gas atom. In all cases, we find that both adsorption sites, top and fcc hollow, are nearly degenerate within vdW-inclusive DFT. Using the PBE $+\mathrm{vdW}^{\text {surf }}$ method, the top adsorption site is energetically favored for $\mathrm{Pd}(111), \mathrm{Cu}(110)$, and $\mathrm{Ag}(111)$ by approximately $5 \mathrm{meV}$ for $\mathrm{Pd}(111)$ and $\mathrm{Ag}(111)$, and $10 \mathrm{meV}$ for $\mathrm{Cu}(110)$. Both adsorption sites are virtually degenerate within our calculation settings in the cases of $\operatorname{Pt}(111)$ and $\mathrm{Cu}(111)$. Figure 2 also displays the contribution to $E_{\text {ads }}$ coming from PBE and vdW interactions upon relaxing the system. More specifically, the PBE contribution destabilizes the fcc hollow adsorption site in the $\mathrm{Cu}$ substrates upon relaxation, as it becomes more positive for both surface orientations. The

TABLE II. Comparison of adsorption energies $E_{\text {ads }}$ between $\mathrm{PBE}+\mathrm{vdW}$ and $\mathrm{PBE}+\mathrm{vdW}$ surf for the top adsorption site of $\mathrm{Xe}$ on transition-metal surfaces. $\mathrm{PBE}+\mathrm{vdW}^{\text {surf }}$ calculations for the fcc hollow adsorption sites are also presented. Experimental data, shown for comparison, are taken from Refs. [37,38,84-96].

\begin{tabular}{lcccc}
\hline \hline & \multicolumn{4}{c}{$E_{\text {ads }}(\mathrm{meV})$} \\
\cline { 2 - 5 } & \multicolumn{2}{c}{ Top } & \multicolumn{2}{c}{ fcc hollow } \\
\cline { 2 - 5 } & PBE+vdW PBE+vdW & \\
\hline $\mathrm{Xerf}$ & $\mathrm{PBE}+\mathrm{vdW}^{\text {surf }}$ & Expt. \\
$\mathrm{Xe} / \mathrm{Pt}(111)$ & -331 & -254 & -253 & -260 to -280 \\
$\mathrm{Xe} / \mathrm{Pd}(111)$ & -325 & -276 & -272 & -310 to -330 \\
$\mathrm{Xe} / \mathrm{Cu}(111)$ & -335 & -248 & -249 & -173 to -200 \\
$\mathrm{Xe} / \mathrm{Ag}(111)$ & -326 & -249 & -239 & -212 to -224 \\
\hline \hline
\end{tabular}

same finding holds for $\operatorname{Ag}(111)$. Although the differences in energy between adsorption sites are too small (a few meV) to regard them as definitive, an accurate determination of exchange and correlation effects (particularly related to vdW interactions) is essential in the structural and energetic features of these systems.

The fact that both adsorption sites for Xe on transition-metal surfaces are nearly degenerate within DFT methods has also been addressed most recently by Chen and co-authors [43], who reported a few meV difference in their PBE and vdW-DF2 calculations between top and fcc hollow adsorption sites. However, they found that results from experiments cannot be explained by energy differences between top and fcc hollow adsorption sites. Instead, by examining the $2 \mathrm{D}$ potential energy surface (PES) of Xe on $\mathrm{Pt}(111)$, they found that the fcc hollow adsorption sites correspond to local maxima in the PES, while top sites correspond to a true minimum. Hence, fcc hollow sites are transient states and thus not easily observed in experiments [40,43]. This result is general, according to their calculations, for the adsorption of noble gases on transition-metal surfaces. They further showed that this fact holds no matter which xc functional is employed. For $\mathrm{Xe} / \mathrm{Pt}(111)$, experimental measurements [91] indeed showed that Xe adsorbs on top sites of the $\operatorname{Pt}(111)$ surface at $T=80 \mathrm{~K}$. Furthermore, measurements also showed that at low coverage, the diffusion barrier for lateral movement of the Xe atoms on the surface is less than $10 \mathrm{meV}$ [97].

Because of the aforementioned reasons, we now discuss the results for the top adsorption site. For comparison, Table II also presents $\mathrm{PBE}+\mathrm{vdW}$ adsorption energies for the top adsorption site as well as the available experimental results. Table II shows that the $\mathrm{PBE}+\mathrm{vdW} \mathrm{W}^{\text {surf }}$ adsorption energies are in very good agreement with experimental results. These calculations slightly underestimate the adsorption energy in the case of $\operatorname{Pt}(111)$ and $\operatorname{Pd}(111)$, while slightly overestimating in the case of both $\mathrm{Cu}$ surfaces and $\operatorname{Ag}(111)$. Nevertheless, these discrepancies amount to approximately $50 \mathrm{meV}$ out of the range of experimental results in the worst case. The $\mathrm{PBE}+\mathrm{vdW}$ method yields larger adsorption energies with respect to $\mathrm{PBE}+\mathrm{vdW} \mathrm{Wurf}^{\text {sur }}$ calculations. This result stems from the freeatom nature of the vdW reference parameters employed in the PBE+vdW method.

Adsorption distances. Table III shows the adsorption distances calculated with the PBE $+\mathrm{vdW}$ and $\mathrm{PBE}+\mathrm{vdW} \mathrm{W}^{\text {surf }}$ methods. The results correspond to the top adsorption site and are reported with respect to the average position of the atoms in the topmost metal layer after relaxation of the system. Experimental results are shown for comparison as well. In general, the calculated adsorption distances with both methods are within $0.10 \AA$ of experimental results except for $\mathrm{Xe} / \mathrm{Cu}(111)$, in which the agreement is within $0.15 \AA$ of the experimental value. We did not find significant differences between PBE+vdW and $\mathrm{PBE}+\mathrm{vdW}$ surf calculations with the exception of Xe on $\mathrm{Cu}(110)$, in which the distance predicted by the PBE+vdW method is $0.12 \AA$ shorter than the $\mathrm{PBE}+\mathrm{vdW}$ surf result. Overall, we find that the $\mathrm{PBE}+\mathrm{vdW}^{\text {surf }}$ results are in closer agreement (within $0.10 \AA$ ) to experimental results than those calculated with other vdW-inclusive DFT methods such as those benchmarked in the work of Chen and co-authors [43]. We note that the experimental adsorption distances that we 
TABLE III. Summary of results for the adsorption of Xe on transition-metal surfaces. These are the equilibrium distances $d_{\mathrm{Xe}-\mathrm{Sub}}$, adsorption energies $E_{\text {ads }}$, and perpendicular vibrational energies $E_{\text {vib }}$ of Xe on top of the surfaces. Experimental results are also displayed for comparison. The distances $d_{\mathrm{Xe}-\mathrm{Sub}}$ are reported with respect to the average distance of the topmost metal layer and correspond to the top adsorption site on each system. Both adsorption distances and energies correspond to the system after relaxation. The values of $d_{\mathrm{Xe}-\mathrm{Sub}}$ and $E_{\text {ads }}$ for $\mathrm{Ag}(111)$ correspond to the best estimates in Ref. [37]. The experimental data are taken from Refs. [37,38,84-96], and [98].

\begin{tabular}{|c|c|c|c|c|c|c|c|c|}
\hline & \multicolumn{3}{|c|}{$d_{\mathrm{Xe}-\mathrm{Sub}}(\AA)$} & \multicolumn{3}{|c|}{$E_{\text {ads }}(\mathrm{meV})$} & \multicolumn{2}{|c|}{$E_{\mathrm{vib}}(\mathrm{meV})$} \\
\hline & $\mathrm{PBE}+\mathrm{vdW}$ & $\mathrm{PBE}+\mathrm{vdW}$ surf & Expt. & $\mathrm{PBE}+\mathrm{vdW}$ & $\mathrm{PBE}+\mathrm{vdW}$ surf & Expt. & $\mathrm{PBE}+\mathrm{vdW}$ surf & Expt. \\
\hline $\mathrm{Xe} / \mathrm{Pt}(111)$ & 3.39 & 3.46 & $3.4 \pm 0.1$ & -331 & -254 & -260 to -280 & 3.9 & $3.5,3.70$ \\
\hline $\mathrm{Xe} / \mathrm{Pd}(111)$ & 3.13 & 3.12 & $3.07 \pm 0.06$ & -325 & -276 & -310 to -330 & 4.2 & \\
\hline $\mathrm{Xe} / \mathrm{Cu}(111)$ & 3.48 & 3.46 & $3.60 \pm 0.08$ & -335 & -248 & -173 to -200 & 3.8 & 2.6 \\
\hline $\mathrm{Xe} / \mathrm{Cu}(110)$ & 3.17 & 3.29 & $3.3 \pm 0.1$ & -326 & -249 & -212 to -224 & 4.0 & $2.5,2.6$ \\
\hline $\mathrm{Xe} / \mathrm{Ag}(111)$ & 3.60 & 3.57 & $3.6 \pm 0.05$ & -244 & -237 & -196 to -226 & 3.8 & 2.79 \\
\hline
\end{tabular}

show in Table III follow the analysis presented by Diehl and co-authors in their review of the topic [38].

Perpendicular vibrational frequencies of Xe. We have also computed the perpendicular vibrational frequencies of $\mathrm{Xe}$ on the metal surfaces to probe the curvature of the potential energy curves around the minimum in each case. For this, we have calculated the adsorption potential energy curve for Xe residing on top sites of each transition-metal surface. We take the case of $\mathrm{Xe}$ on $\mathrm{Pt}(111)$ as an example: Figure 3 shows its adsorption potential energy $E_{\text {ads }}$ as a function of vertical distance $d$ of the Xe monolayer employing the PBE and the $\mathrm{PBE}+\mathrm{vdW}$ surf methods. The adsorption energy per adsorbed atom was calculated using Eq. (34) where the unrelaxed system was employed for all the calculated points. The vertical distance $d$ was defined as the difference of the position of the atom in the monolayer with respect to the position of the unrelaxed topmost metallic layer. The experimental adsorption distance and energy are displayed in blue shaded regions. The adsorption distance and energy observed in the potential curve of Fig. 3 agree very well with the ones presented in Table III,

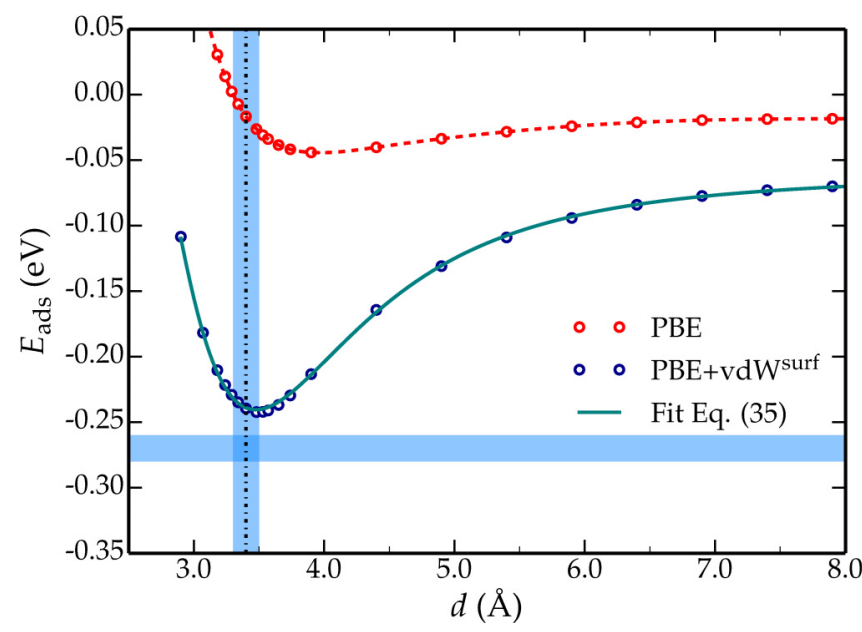

FIG. 3. Potential-energy curve as a function of vertical distance $d$ of a Xe monolayer on top of $\mathrm{Pt}(111)$ with different approximations within DFT. The blue shaded regions correspond to the experimental adsorption distance [91] of $3.4 \pm 0.1 \AA$ and to the interval of experimental adsorption energy [38] that ranges from -260 to -280 meV. which shows a summary of the PBE $+v d W^{\text {surf }}$ results for Xe on metal surfaces after relaxing each system. This fact holds also for the case of the other metal surfaces here studied. It shows that, in the case of Xe on metal surfaces, the adsorption potential-energy curves provide relevant information on the adsorption process. Based on them, we have calculated the perpendicular vibrational energy of $\mathrm{Xe}$ in each adsorption case. We note that, unlike our own previous work [22], we have included vdW interactions between metal atoms in the adsorption energies of each potential-energy curve.

Following previous works $[40,42,43]$, we have modeled the gas-surface adsorption potential with the following function given by the sum of repulsive and attractive vdW interactions:

$$
E(d)=\alpha_{1} e^{-\alpha_{2} d}-\frac{C_{3}}{\left(d-Z_{0}\right)^{3}}+E_{\mathrm{ml}},
$$

where $E(d)$ is the adsorption potential between $\mathrm{Xe}$ and the metal substrate at a distance $d$ from the surface and $E_{\mathrm{ml}}$ is a constant that corresponds approximately to the formation energy of the Xe monolayer. We have determined the parameters $\alpha_{1}, \alpha_{2}, C_{3}, Z_{0}$, and $E_{\mathrm{ml}}$ by fitting Eq. (35) to the $\mathrm{PBE}+\mathrm{vdW}$ surf calculations. The resulting curve of the fit is depicted with a solid line in Fig. 3 for the case of Xe on $\operatorname{Pt}(111)$. The vibrational energy $E_{\text {vib }}$ is then given by

$$
E_{\mathrm{vib}}=h v=\frac{h}{2 \pi} \sqrt{\frac{k_{e}}{m_{\mathrm{Xe}}}},
$$

where $v, h$, and $m_{\mathrm{Xe}}$ are the vibrational frequency, Planck's constant, and the mass of an atom of Xe, respectively. The force constant $k_{e}$ corresponds to the second derivative evaluated at the minimum of the potential given by Eq. (35). Following this procedure, the results for $E_{\mathrm{vib}}$ are given in Table III.

To the best of our knowledge, experimental measurements for the perpendicular vibrational energy exist for $\mathrm{Xe} / \mathrm{Pt}(111), \mathrm{Xe} / \mathrm{Cu}(111), \mathrm{Xe} / \mathrm{Cu}(110)$, and $\mathrm{Xe} / \mathrm{Ag}(111)$. In the case of $\mathrm{Pt}(111)$, the values of 3.5 and $3.70 \mathrm{meV}$ have been reported $[92,93]$. The $\mathrm{PBE}+\mathrm{vdW}$ surf calculations yield a perpendicular vibrational energy of $3.9 \mathrm{meV}$ which is in fair agreement with experimental results. In the cases of $\mathrm{Cu}(111), \mathrm{Cu}(110)$, and $\mathrm{Ag}(111)$, the $\mathrm{PBE}+\mathrm{vdW}^{\text {surf }}$ values overestimate the experimental values $[94,96,98]$ by $1.2,1.4$, and $1.0 \mathrm{meV}$, respectively. With the exception of $\operatorname{Pt}(111)$, the results show that the $\mathrm{PBE}+\mathrm{vdW} \mathrm{W}^{\text {surf }}$ method overestimates the curvature around the minimum, yielding higher 
perpendicular vibrational energies by approximately $1.2 \mathrm{meV}$ with respect to experiment and other vdW-inclusive DFT methods such as vdW-DF2 [43]. However, as it has been observed by Carrasco and co-workers [99], the vdW-DF2 method yields $C_{3}$ coefficients that are approximately two times smaller than those obtained in methods such as the $\mathrm{PBE}+\mathrm{vdW} \mathrm{W}^{\text {surf }}$ or (the empirically optimized vdW-DF-type functional) optB88-vdW [14]. The overestimation of the curvature with the $\mathrm{PBE}+\mathrm{vdW}$ surf method is probably related to a slight overestimation of the adsorption energy with the $\mathrm{PBE}+\mathrm{vdW} \mathrm{W}^{\text {surf }}$ due to the absence of many-body dispersion effects as we have observed in recent work [100].

We present a summary of the $\mathrm{PBE}+\mathrm{vdW} \mathrm{W}^{\text {surf }}$ results for Xe on metal surfaces in Table III. The noticeable agreement of both adsorption distances and energies with respect to experimental values indicates the importance of the inclusion of the nonlocal collective effects present in the surface when calculating vdW interactions. With the exception of the vibrational energies, we find that the $\mathrm{PBE}+\mathrm{vdW}^{\text {surf }}$ results are in closer agreement to experimental results than those calculated with other vdW-inclusive DFT methods such as the ones benchmarked in the work of Chen and co-authors [43].
We remark that accurate results might also be achieved by vdW-DF-type functionals with empirically optimized exchange [14,27,99].

In spite of the essential difference between the $\mathrm{PBE}+\mathrm{vdW} \mathrm{Wurf}^{\text {sund }} \mathrm{PBE}+\mathrm{vdW}$ methods, our calculations show that the $\mathrm{PBE}+\mathrm{vdW}$ adsorption distances are also in very good agreement with experimental results (see Table III) in the case of the adsorption of $\mathrm{Xe}$ on transition-metal surfaces. Regardless of this agreement, the PBE+vdW scheme leads to an overestimation of the adsorption energy as the input $\mathrm{vdW}$ parameters for the metal atom correspond to the free atom, neglecting the effects of the collective response of the solid (see Table I). Of particular relevance is the fact, which we have observed above, that neglecting the environmental effects of the solid in the determination of the vdW parameters can lead to inaccurate equilibrium structures and an overestimation of the binding strength with respect to experiments in more complex systems such as organic/inorganic interfaces [22]. In this context, in the next section we analyze the adsorption potential of a single planar molecule of 3,4,9,10-perylenetetracarboxylic acid dianhydride $\left(\mathrm{C}_{24} \mathrm{H}_{8} \mathrm{O}_{6}, \mathrm{PTCDA}\right)$ on a $\mathrm{Au}(111)$ surface. (a) PTCDA<smiles>O=C1OC(=O)c2ccc3c4ccc5c6c(ccc(c7ccc1c2c73)c64)C(=O)OC5=O</smiles>

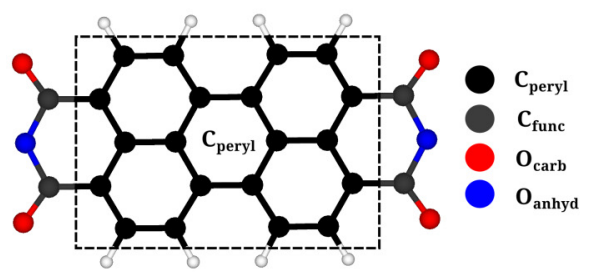

(c) PTCDA on Ag(100)

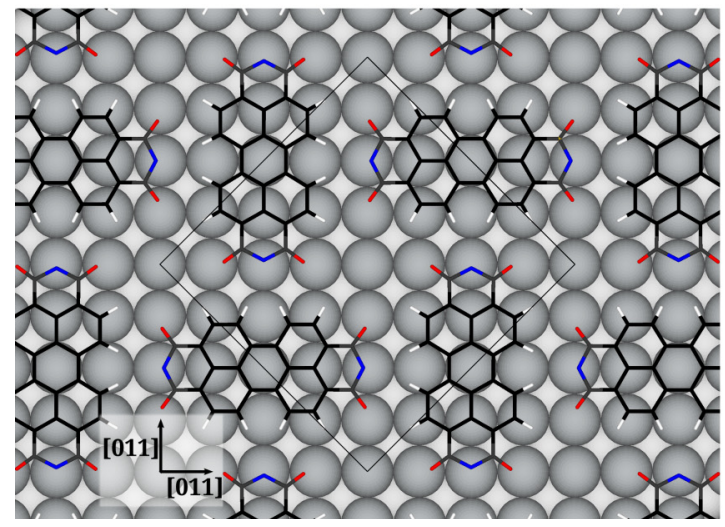

\section{(b) PTCDA on Ag(111)}

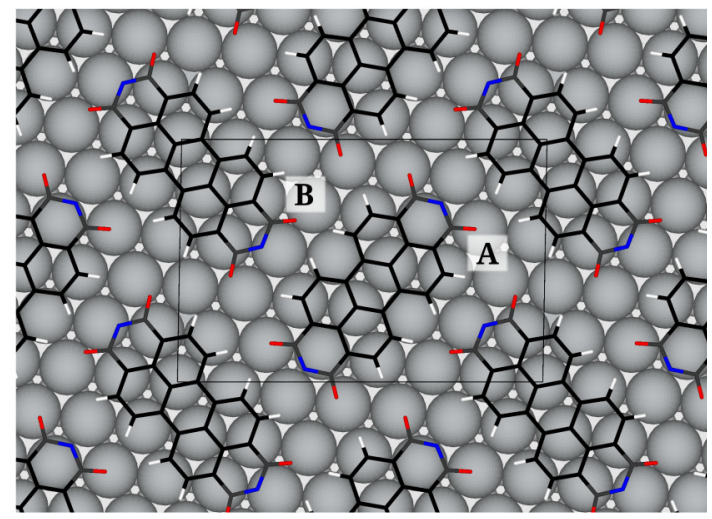

(d) PTCDA on Ag(110)

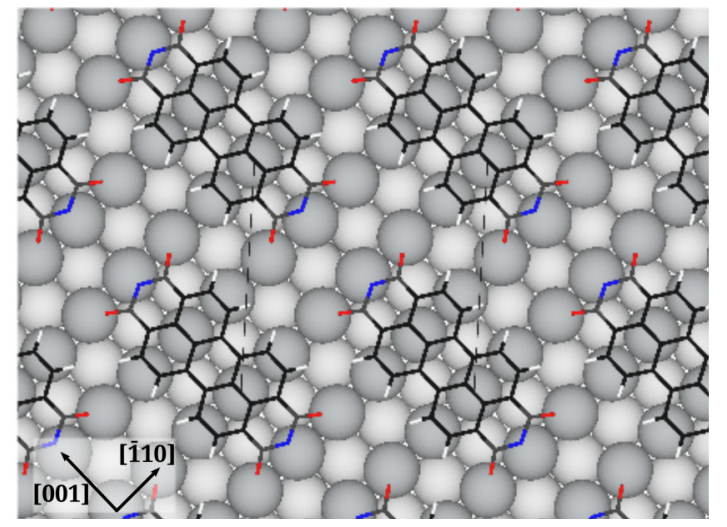

FIG. 4. (a) Chemical structure of PTCDA. The distinction between carbon atoms belonging to the perylene core $\left(\mathrm{C}_{\text {peryl }}\right.$, black) and to the functional groups $\left(\mathrm{C}_{\text {funct }}\right.$, dark gray) is also displayed. In a similar fashion, oxygen atoms are shown in red for the case of the carboxylic oxygen $\left(\mathrm{O}_{\text {carb }}\right)$ and blue for the anhydride oxygen $\left(\mathrm{O}_{\text {anhyd }}\right)$. (b) Top view of the relaxed structure of PTCDA on $\mathrm{Ag}(111)$. Both inequivalent molecules of the structure are labeled A and B. (c) Top view of the relaxed structure of PTCDA on Ag(100). (d) Top view of the relaxed structure of PTCDA on $\operatorname{Ag}(110)$. The topmost metal layer is displayed in dark gray while the sublayer is light gray. Images of the structures were produced using the visualization software VESTA [106]. 

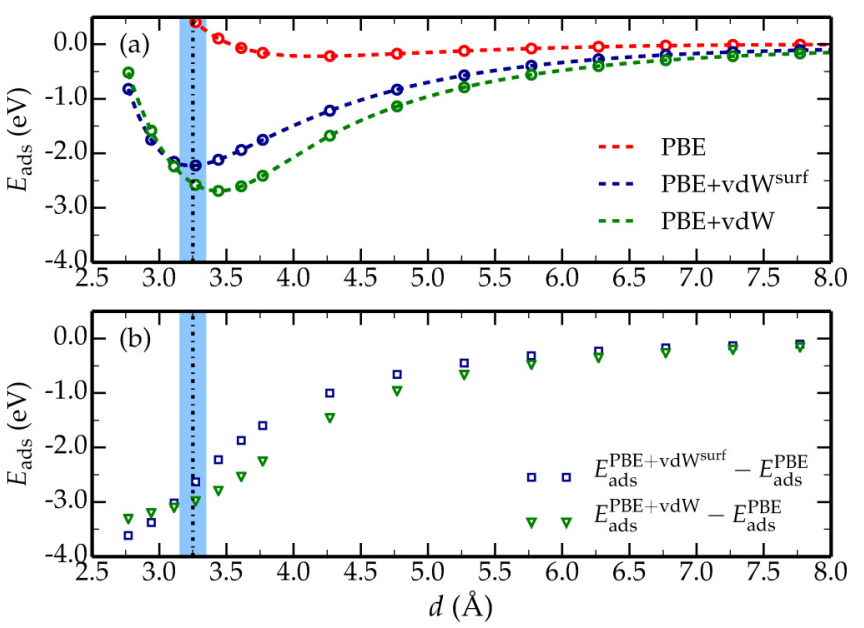

FIG. 5. (a) Adsorption energy $E_{\text {ads }}$ as a function of vertical distance $d$ for PTCDA on $\mathrm{Au}(111)$. The distance $d$ is evaluated with respect to the position of the unrelaxed topmost metal layer. The blue shaded region corresponds to the experimental adsorption distance of $3.25 \pm 0.1 \AA$ as determined by Wagner and co-workers [79]. The error bar corresponds to typical experimental error estimates. (b) Contribution of vdW interactions to the adsorption energy as a function of vertical distance $d$ for PTCDA on $\mathrm{Au}(111)$, which is defined as the difference between either the $\mathrm{PBE}+\mathrm{vdW} \mathrm{wurf}^{\text {sur }}$ or the $\mathrm{PBE}+\mathrm{vdW}$ energy and the PBE energy.

\section{PTCDA on Au(111)}

PTCDA is a chemical compound formed by an aromatic perylene core $\left(\mathrm{C}_{\text {peryl }}\right)$ terminated with two anhydride functional groups, each of them containing two carbon atoms $\left(\mathrm{C}_{\text {func }}\right)$, two carboxylic oxygens $\left(\mathrm{O}_{\text {carb }}\right)$, and one anhydride oxygen $\left(\mathrm{O}_{\text {anhyd }}\right)$ [see Fig. 4(a) and Ref. [44]]. Experimental studies observe that PTCDA is physisorbed on Au(111), hence its bonding interaction is governed mainly by vdW forces [79,101-103]. Wagner and co-workers [79] studied the system based on single-molecule manipulation experiments. By combining scanning tunneling microscopy and frequencymodulated atomic force microscopy, they reported an adsorption energy of about $-2.5 \mathrm{eV}$ per molecule of PTCDA and an adsorption distance of approximately $3.25 \AA$, a value which is displayed as a blue shaded region in Fig. 5. The case of PTCDA on $\mathrm{Au}(111)$ has also been measured using the normal incidence $\mathrm{X}$-ray standing wave (NIXSW) technique by Henze and coauthors [101,104], where they found an adsorption distance of $3.31 \AA$ for the PTCDA monolayer. In addition, temperatureprogramed desorption (TPD) experiments performed to study the adsorption of the monolayer reveal an adsorption energy of approximately $-1.94 \mathrm{eV}$ per molecule [105]. For these reasons and the experimental information that is available, PTCDA on $\mathrm{Au}(111)$ serves as an interesting example of physisorption in an organic/inorganic interface.

Adsorption potential curve. We have calculated the adsorption potential curve of a single PTCDA molecule on $\mathrm{Au}(111)$ using the PBE, PBE+vdW, and PBE+vdW ${ }^{\text {surf }}$ schemes, which are displayed in Fig. 5(a). The adsorption energy per adsorbed molecule was calculated using Eq. (34) where $E_{\text {AdSys }}$ is the total energy of the adsorption system (PTCDA + metallic surface), $E_{\mathrm{Me}}$ is the energy of the bare metal slab, and $E_{\text {Ad }}$ is the energy of a single PTCDA molecule in periodic boundary conditions. The calculations correspond to the unrelaxed system where the metal slab was generated using the experimental lattice constant of $\mathrm{Au}$ in order to have a direct comparison with experimental results. The potential-energy curve was calculated including vdW interactions between metal atoms. From Fig. 5(a), it is clear that the PBE functional cannot accurately describe the adsorbate-substrate interaction as it leads to an exceptionally small adsorption energy. Both $\mathrm{PBE}+\mathrm{vdW}$ and $\mathrm{PBE}+\mathrm{vdW}^{\text {surf }}$ calculations show a stronger interaction due to the inclusion of vdW interactions. Figure 5(a) shows that a single molecule of PTCDA adsorbs at a height of approximately $3.44 \AA$ with an adsorption energy of approximately $-2.69 \mathrm{eV}$ per molecule with the PBE+vdW method. On the other hand, the PBE+vdW ${ }^{\text {surf }}$ calculation leads to an adsorption distance of approximately $3.23 \AA$ and an adsorption energy of approximately $-2.23 \mathrm{eV}$ per molecule. We have performed the same calculation using the PBE lattice constant of Au (4.159 $\AA$, in accordance to our previous work in Ref. [29]) to generate the surface slab. The adsorption potential curve yields in this way an adsorption distance of $3.21 \AA$ and an adsorption energy of $-2.17 \mathrm{eV}$ with the $\mathrm{PBE}+\mathrm{vdW}^{\text {surf }}$ method. These values do not change considerably with respect to the lattice constant used so we proceed now to compare the values that we obtain when the experimental lattice constant is used.

In contrast to the adsorption of Xe on metal surfaces, we observe differences in the adsorption distance predicted by both methods, resulting in a larger distance with the PBE + vdW method by $0.21 \AA$ with respect to its $\mathrm{PBE}+\mathrm{vdW} \mathrm{W}^{\text {surf }}$ counterpart. The $\mathrm{PBE}+\mathrm{vdW}$ adsorption distance result is overestimated if we consider the values of 3.25 and $3.31 \AA$ found in experiments for the single molecule and monolayer, respectively [79,101]. On the other hand, $\mathrm{PBE}+\mathrm{vdW} \mathrm{W}^{\text {surf }}$ calculations lead to remarkable agreement with experiments. With respect to the binding strength, the PBE+vdW adsorption energy also seems overestimated with respect to the experimental values $[79,105]$ of -2.5 and $-1.9 \mathrm{eV}$ measured for the single molecule and monolayer, respectively. Regarding the $\mathrm{PBE}+\mathrm{vdW}$ surf adsorption energy for the single molecule, its value of $-2.23 \mathrm{eV}$ lies in-between these two experimental results.

The effect of dielectric screening in the $v d W$ parameters. The differences in these results reflect the impact of the vdW parameters on the accuracy when it comes to the structure of organic/inorganic interfaces. In the $\mathrm{PBE}+\mathrm{vdW}$ surf method, the set of reduced $C_{6}$ coefficients that are determined by the dielectric screening of the bulk yields a smaller vdW energy. We show this effect in Fig. 5(b) where we display the contribution of $\mathrm{vdW}$ interactions to the adsorption potential curve, showing how the reduced $C_{6}$ coefficient of Au yields a smaller vdW energy in the PBE $+v_{d W}{ }^{\text {surf }}$ method. This feature modifies the adsorption potential in a nontrivial manner, with particular relevance at the range of the adsorption distance. In addition, the coefficients are effectively changed by the electronic environment of each atom reflecting the interface polarization due to local hybridization effects. Taking PTCDA on $\mathrm{Au}(111)$ as an example, Fig. 6(a) demonstrates how the $C_{6}$ coefficient between a $\mathrm{C}$ atom of the adsorbate molecule 


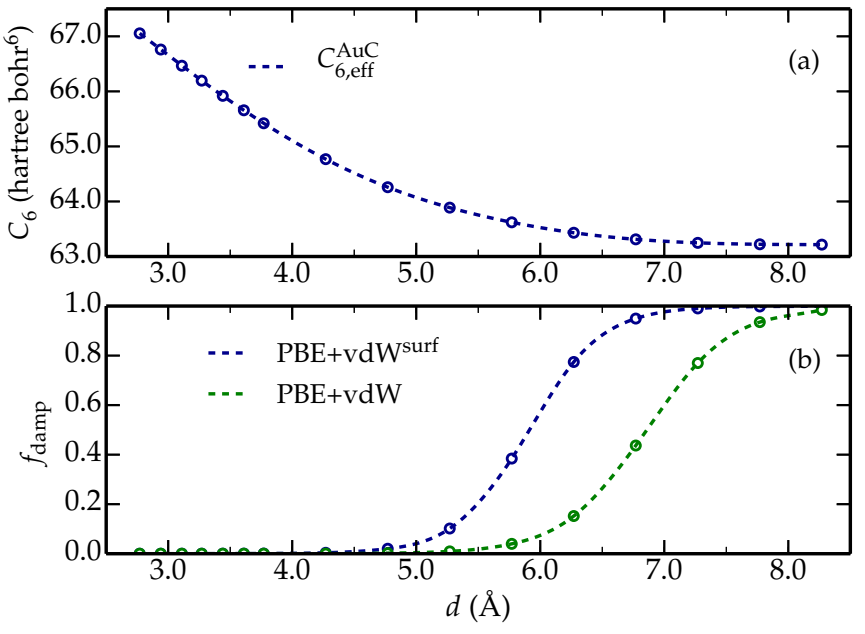

FIG. 6. (a) Changes in the $C_{6}^{\mathrm{AuC}}$ coefficient with respect to the adsorption distance $d$ for a single PTCDA molecule on $\mathrm{Au}(111)$ calculated with the PBE+vdW ${ }^{\text {surf }}$ method. (b) Variations of the damping function $f_{\text {damp }}$ with respect to the adsorption distance $d$ when using the PBE $+\mathrm{vdW}$ and $\mathrm{PBE}+\mathrm{vdW}{ }^{\text {surf }}$ methods. The onset of $f_{\text {damp }}$ occurs at a smaller distance in the PBE $+v_{d W} W^{\text {surf }}$ method.

and a $\mathrm{Au}$ atom of the surface changes with the adsorption distance $d$. The changes occurring at each distance are a consequence of variations in the electronic environment of each atom. Furthermore, the reduction of the vdW radii in the surface atoms leads to a larger relative weight of $\mathrm{vdW}$ contributions at shorter range with the $\mathrm{PBE}+\mathrm{vdW} \mathrm{Wurf}^{\text {surf }}$ method in comparison to the PBE+vdW method. This can be observed in Fig. 6(b) as the onset of the damping function $f_{\text {damp }}$ in the $\mathrm{PBE}+\mathrm{vdW} \mathrm{Wurf}^{\text {sur }}$ method occurs at smaller adsorption distances due to the reduced $\mathrm{vdW}$ radius of $\mathrm{Au}$. The coaction of the effects in the $C_{6}$ coefficients and the vdW radii causes a nontrivial reduction of both the adsorption energy and the adsorption distance, which modifies the potential-energy curve of PTCDA on $\mathrm{Au}(111)$ as observed in Fig. 5.

\section{Comparison between close-packed and non-close-packed surfaces}

Previously, we have analyzed the performance of the PBE $+v_{d W}{ }^{\text {surf }}$ method in the case of the adsorption on closepacked (111) surfaces of some transition metals. Here, we analyze the case of non-close-packed surfaces. We compare the case of a Xe monolayer on the $\mathrm{Cu}(110)$ and $\mathrm{Cu}(111)$ surfaces and the adsorption of an organic adsorbate on a metallic surface with different orientations: the adsorption of a PTCDA monolayer on the $\operatorname{Ag}(111), \operatorname{Ag}(100)$, and $\operatorname{Ag}(110)$ surfaces.

Xe on $\mathrm{Cu}(110)$ and $\mathrm{Cu}(111)$. The adsorption potential of $\mathrm{Xe}$ on $\mathrm{Cu}(110)$ is depicted in Fig. 7. In the blue shaded region, the adsorption distance of $3.3 \pm 0.1 \AA$ is shown as measured by Caragiu and co-workers [85] using LEED. The excellent agreement of the $\mathrm{PBE}+\mathrm{vdW}$ surf equilibrium distance with the experimental result is evident from Fig. 7.

The vdW parameters used as an input for the $\mathrm{PBE}+\mathrm{vdW}$ surf method are calculated according to the dielectric function of the bulk material. As a consequence, the input parameters

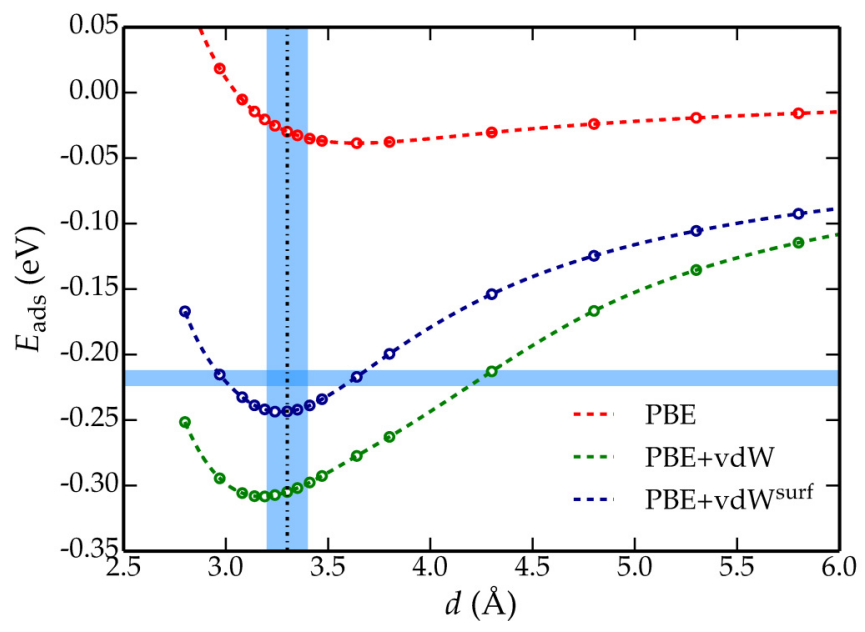

FIG. 7. Potential-energy curve as a function of vertical distance $d$ of a Xe monolayer on top of $\mathrm{Cu}(110)$ with different approximations within DFT. The blue shaded regions correspond to the experimental adsorption distance [85] of $3.3 \pm 0.1 \AA$ and experimental adsorption energy [86] of $-218 \pm 6 \mathrm{meV}$ (see also Table III).

for a given surface are the same no matter the surface termination. The method relies on the differences in the electronic environment given as a result of different surface terminations, which are reflected in the evaluation of the $\mathrm{vdW}$ parameters based on the Hirshfeld partitioning scheme [see Eqs. (30)-(32)]. As an example of this, Fig. 8(a) shows the effective $C_{6}$ parameters for the interaction between $\mathrm{Xe}$ and $\mathrm{Cu}$ as calculated in the PBE $+\mathrm{vdW}$ surf method for the adsorption of a Xe monolayer on both the $\mathrm{Cu}(110)$ and $\mathrm{Cu}(111)$ surfaces. It
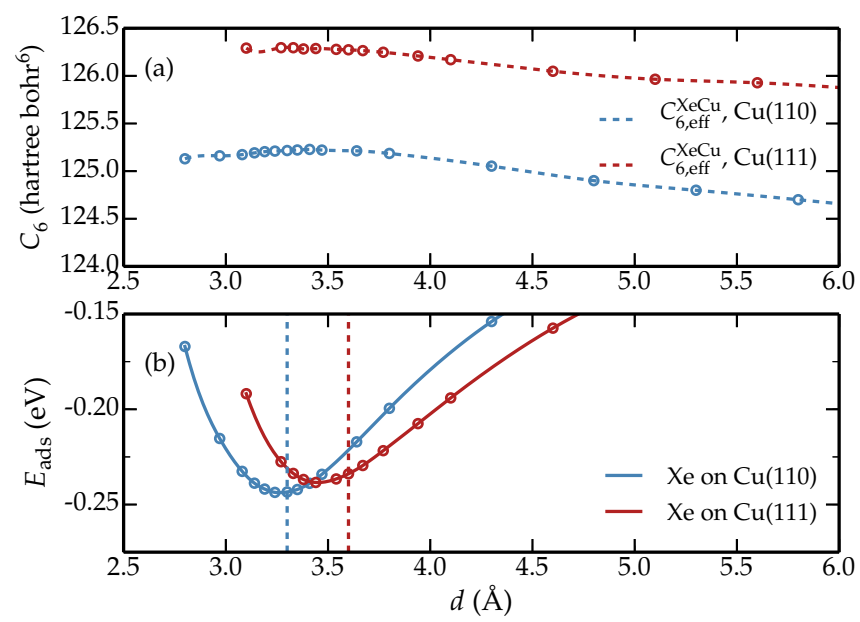

FIG. 8. (a) Changes in the $C_{6}^{\mathrm{XeCu}}$ coefficient with respect to the adsorption distance $d$ for $\mathrm{Xe}$ on $\mathrm{Cu}(110)$ (blue) and $\mathrm{Cu}(111)$ (red) calculated with the $\mathrm{PBE}+\mathrm{vdW}^{\text {surf }}$ method. (b) Potential-energy curve as a function of vertical distance $d$ of $\mathrm{Xe}$ on top of $\mathrm{Cu}(110)$ (blue) and $\mathrm{Cu}(111)$ (red) calculated with the PBE+vdW ${ }^{\text {surf }}$ method. The blue dashed line corresponds to the experimental adsorption distance [85] of $3.3 \pm 0.1 \AA$ for $\mathrm{Xe}$ on $\mathrm{Cu}(110)$. The red dashed line corresponds to the experimental adsorption distance [84] of $3.60 \pm 0.08 \AA$ for $\mathrm{Xe}$ on $\mathrm{Cu}(111)$. 
TABLE IV. Comparison of experimental and theoretical results for the adsorption geometry of PTCDA on $\operatorname{Ag}(111), \operatorname{Ag}(100), \operatorname{and} \operatorname{Ag}(110)$. We use $d_{\mathrm{Th} / \mathrm{Expt}}$ to denote the averaged vertical adsorption heights of the specific atoms obtained from PBE+vdW ${ }^{\text {surf }}$ calculations and NIXSW studies. The adsorption height is given in $\AA$ with respect to the topmost unrelaxed metal layer. The specification of the atoms can be seen in Fig. 4(a). The $\mathrm{C}$ backbone distortion is given as $\Delta \mathrm{C}=d\left(\mathrm{C}_{\text {peryl }}\right)-d\left(\mathrm{C}_{\text {func }}\right)$ and the $\mathrm{O}$ difference as $\Delta \mathrm{O}=d\left(\mathrm{O}_{\text {anhyd }}\right)-d\left(\mathrm{O}_{\text {carb }}\right)$. Experimental results can be found in Refs. [44,45,104]. We cite here the results given in Refs. [44,104].

\begin{tabular}{|c|c|c|c|c|c|c|}
\hline & \multicolumn{2}{|c|}{$\operatorname{Ag}(111)$} & \multicolumn{2}{|c|}{$\operatorname{Ag}(100)$} & \multicolumn{2}{|c|}{$\operatorname{Ag}(110)$} \\
\hline & $d_{\mathrm{Th}}$ & $d_{\text {Expt }}[104]$ & $d_{\mathrm{Th}}$ & $d_{\text {Expt }}[44]$ & $d_{\mathrm{Th}}$ & $d_{\text {Expt }}[44]$ \\
\hline $\mathrm{C}$ total & 2.80 & $2.86 \pm 0.01$ & 2.75 & $2.81 \pm 0.02$ & 2.54 & $2.56 \pm 0.01$ \\
\hline $\mathrm{C}_{\text {peryl }}$ & 2.80 & & 2.76 & $2.84 \pm 0.02$ & 2.56 & $2.58 \pm 0.01$ \\
\hline $\mathrm{C}_{\text {func }}$ & 2.78 & & 2.67 & $2.73 \pm 0.01$ & 2.43 & $2.45 \pm 0.11$ \\
\hline$\Delta \mathrm{C}$ & 0.02 & & 0.09 & $0.11 \pm 0.02$ & 0.13 & $0.13 \pm 0.11$ \\
\hline O total & 2.73 & $2.86 \pm 0.02$ & 2.59 & $2.64 \pm 0.02$ & 2.33 & $2.33 \pm 0.03$ \\
\hline $\mathrm{O}_{\text {carb }}$ & 2.68 & $2.66 \pm 0.03$ & 2.54 & $2.53 \pm 0.02$ & 2.29 & $2.30 \pm 0.04$ \\
\hline $\mathrm{O}_{\text {anhyd }}$ & 2.83 & $2.98 \pm 0.08$ & 2.69 & $2.78 \pm 0.02$ & 2.39 & $2.38 \pm 0.03$ \\
\hline$\Delta \mathrm{O}$ & 0.15 & $0.32 \pm 0.09$ & 0.15 & $0.25 \pm 0.02$ & 0.10 & $0.08 \pm 0.05$ \\
\hline
\end{tabular}

displays the variations in the average effective $C_{6}$ coefficient between $\mathrm{Xe}$ and the topmost $\mathrm{Cu}$ layer as the distance $d$ is changed. The differences in the electronic environments result in different values for the $C_{6}$ coefficients, which give rise to surface termination sensitivity. This has a contribution in the adsorption potentials shown in Fig. 8(b) and the adsorption distances reported in Table III. It is worth mentioning that greater differences are found in more complex systems such as the case of organic/inorganic interfaces. For instance, Al-Saidi and co-workers [107] found significant surface sensitivity in the adsorption of 2-pyrrolidone on $\operatorname{Ag}(111)$ and $\operatorname{Ag}(100)$ which was better understood when including vdW interactions with the PBE+vdW ${ }^{\text {surf }}$ method.

\section{PTCDA on Ag surfaces}

We address now the case of an organic adsorbate on a metallic surface with different orientations: the adsorption of PTCDA on $\mathrm{Ag}(111), \operatorname{Ag}(100)$, and $\mathrm{Ag}(110)$. We performed a structural optimization of each of these systems where the molecule and the atoms in the topmost two metal layers were allowed to relax using the $\mathrm{PBE}+\mathrm{vdW} \mathrm{Wrr}^{\text {surf }}$ method. We have taken the experimental configuration as starting point for each structural optimization. The adsorption geometries of these systems have been investigated using the NIXSW technique $[44,45,104]$. A novel feature in the studies including PTCDA on $\operatorname{Ag}(100)$ and $\operatorname{Ag}(110)$ is their higher chemical resolution resulting in the extraction of the adsorption positions of each of the chemically inequivalent atoms in PTCDA. We compare these results with $\mathrm{PBE}+\mathrm{vdW} \mathrm{Wurf}^{\text {sulculations in }}$ Table IV and illustrate them in Fig. 9, in which the adsorption position of each of the atoms is referred to the position of the topmost unrelaxed metal layer.

PTCDA forms a commensurate monolayer structure on silver surfaces. On $\mathrm{Ag}(111)$, it forms a herringbone structure with two molecules per unit cell in nonequivalent adsorption configurations [82,108]. Both molecules are adsorbed on bridge position, molecule $\mathrm{A}$ is practically aligned with the substrate in the [101] direction with its carboxylic oxygen atoms on top position and the anhydride oxygen atoms located on bridge sites. Molecule B on the other hand is rotated with respect to the [011] direction, with most atoms in its functional groups located closely to adsorption bridge positions [44,108]. Figure 4(b) depicts how this configuration is well reproduced in our calculations after relaxing the system. On $\mathrm{Ag}(100)$, a T-shape arrangement with two adsorbed molecules per unit cell can be observed [83]. Figure 4(c) shows the top view of the system after relaxation, showing that both molecules are aligned with the [110] direction of the substrate with the center of each molecule adsorbed on top position. This result agrees very well with experiments and previous DFT calculations [44]. Finally, in the case of $\operatorname{Ag}(110)$, PTCDA forms a brick-wall adsorption pattern with one molecule adsorbed per surface unit cell [82]. The long axis of the molecule is located parallel to the [001] direction, while the center of the molecule is located on the bridge site between the close-packed atomic rows parallel to the [1110] direction [109]. This configuration is reproduced accurately by the PBE+vdW ${ }^{\text {surf }}$ calculations as Fig. 4(d) confirms.

Table IV shows that the $\mathrm{PBE}+\mathrm{vdW}$ surf results for the vertical adsorption distance agree very well with experimental results. With the exception of the anhydride oxygen in $\mathrm{Ag}(111)$, the calculated distances for all atoms that form the molecule lie within $0.1 \AA$ of the experimental results for all three surfaces. These results also reveal that our calculations reproduce the experimental trends observed in the sequence of $\operatorname{Ag}(111), \operatorname{Ag}(100)$, and $\operatorname{Ag}(110)$ [44,45]. The overall vertical adsorption height, taken as an average over all carbon atoms, given by the calculations decreases in the sequence by $0.26 \AA$, in comparison to the value of $0.30 \AA$ obtained in experiments. The calculations reproduce the transition from a saddlelike adsorption geometry of PTCDA on $\operatorname{Ag}(111)$ to the archlike adsorption geometry that can be found in the more open surfaces according to experiments (see Fig. 9). Finally, for the above-mentioned sequence we find an increase in the $\mathrm{C}$ backbone distortion $\Delta \mathrm{C}=d\left(\mathrm{C}_{\text {peryl }}\right)-d\left(\mathrm{C}_{\text {func }}\right)$ and a decrease in the $\mathrm{O}$ difference $\Delta \mathrm{O}=d\left(\mathrm{O}_{\text {anhyd }}\right)-d\left(\mathrm{O}_{\text {carb }}\right)$. For $\Delta \mathrm{C}$, the calculations yield $0.02,0.09$, and $0.13 \AA$ for $\operatorname{Ag}(111), \operatorname{Ag}(100)$, and $\operatorname{Ag}(110)$, respectively, values which are in excellent agreement with experiments $[44,45]$. In the case of $\mathrm{Ag}(111)$, the $\mathrm{C}$ backbone distortion has not been determined experimentally [104], but the saddlelike adsorption geometry suggests a minimum distortion of the $\mathrm{C}$ backbone $[44,104]$ which we observe in our calculations as well. The $\mathrm{C}$ backbone 
(a) PTCDA on Ag(111)

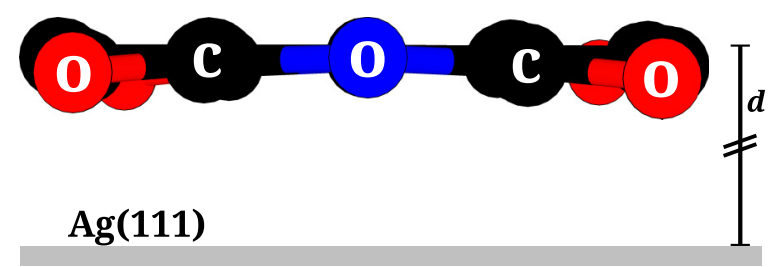

(b) PTCDA on Ag(100)

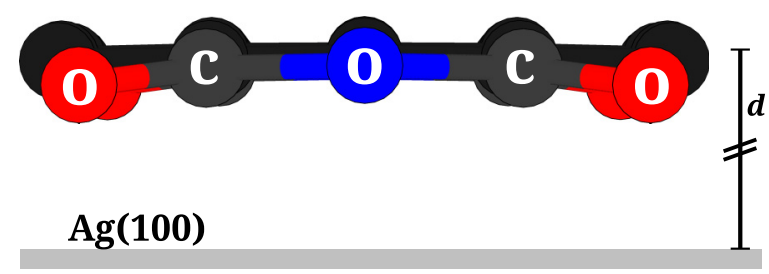

(c) PTCDA on Ag(110)

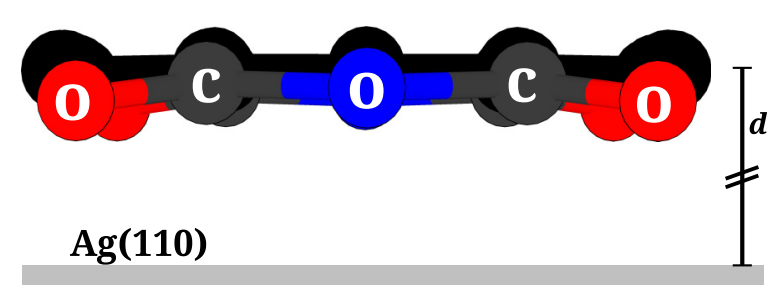

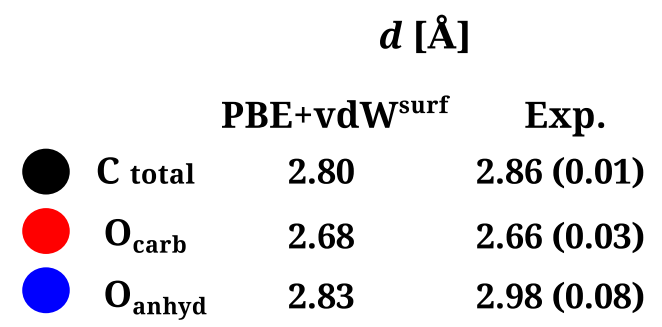

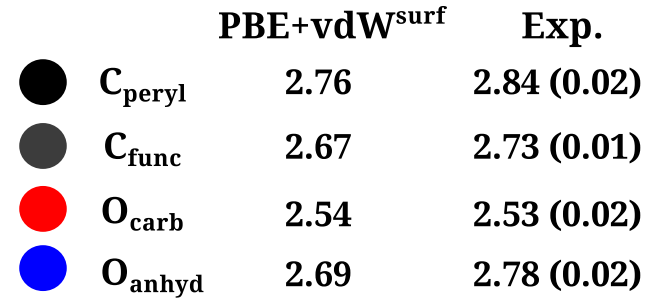

\begin{tabular}{|c|c|c|}
\hline & PBE+vdW $W^{\text {surf }}$ & Exp. \\
\hline$C_{\text {peryl }}$ & 2.56 & $2.58(0.01)$ \\
\hline $\mathrm{C}_{\text {func }}$ & 2.43 & $2.45(0.11)$ \\
\hline $\mathbf{O}_{\text {carb }}$ & 2.29 & $2.30(0.04)$ \\
\hline $\mathbf{O}_{\text {anhyd }}$ & 2.39 & $2.38(0.03)$ \\
\hline
\end{tabular}

FIG. 9. Geometry of PTCDA when adsorbed on (a) $\operatorname{Ag}(111)$, (b) $\operatorname{Ag}(100)$, and (c) $\operatorname{Ag}(110)$. The equilibrium distances $d$ for each chemically inequivalent atom calculated with the PBE $+\mathrm{vdW}^{\text {surf }}$ method are displayed. Experimental results [44] from NIXSW studies are also shown for comparison. The distinction between carbon atoms belonging to the perylene core $\left(\mathrm{C}_{\text {peryl }}\right.$, black $)$ and to the functional groups $\left(\mathrm{C}_{\text {funct }}\right.$, dark gray) is also displayed. In a similar fashion, oxygen atoms are shown in red for the case of the carboxylic oxygen $\left(\mathrm{O}_{\text {carb }}\right)$ and blue for the anhydride oxygen $\left(\mathrm{O}_{\text {anhyd }}\right)$. Images of the structures were produced using the visualization software VESTA [106].

distortion in $\operatorname{Ag}(100)$ and $\operatorname{Ag}(110)$ is then remarkably well reproduced by the calculations.

With respect to the oxygen difference $(\Delta \mathrm{O})$, the resulting values are $0.15 \AA$ for $\operatorname{Ag}(111)$ and $\operatorname{Ag}(100)$, and $0.10 \AA$ for $\mathrm{Ag}(110)$. These values reproduce the decrease in the sequence observed by experiments but underestimate the difference by $0.17 \AA$ in $\operatorname{Ag}(111)$ and $0.10 \AA$ in $\operatorname{Ag}(100)$. This underestimation lies in the fact that the adsorption distances for the anhydride oxygen obtained with the calculations are also underestimated in the cases of $\operatorname{Ag}(111)$ and $\operatorname{Ag}(100)$. On the other hand, the calculated distance for the anhydride oxygen in $\mathrm{Ag}(110)$ agrees very well with experiments, leading to a very good agreement with the experimental result of $0.08 \pm 0.05 \AA$ in the oxygen difference [44].

We have also computed the adsorption energy $E_{\text {ads }}$ per molecule of the systems using Eq. (34) where $E_{\text {AdSys }}$ is the total energy of the adsorption system (PTCDA + metallic surface), $E_{\mathrm{Me}}$ is the energy of the bare metal slab, $E_{\mathrm{Ad}}$ is the energy of the PTCDA monolayer in periodic boundary conditions, and the final energy is divided by two in the cases in which the monolayer consists of two molecules. All quantities are taken after relaxation of each subsystem; we summarize the results in Table V. The binding strength increases in the abovementioned sequence, yielding the values of $-2.86,-2.93$, and
-3.39 eV for $\mathrm{Ag}(111), \mathrm{Ag}(100)$, and $\mathrm{Ag}(110)$, respectively. The vdW interactions are essential in these systems as they are the larger contribution to the adsorption energy, representing $73 \%$ for $\mathrm{Ag}(110)$ and the only stabilizing energy in $\mathrm{Ag}(111)$ and $\mathrm{Ag}(100)$. The chemical interactions become only relevant in $\operatorname{Ag}(111)$ and $\operatorname{Ag}(110)$. Only in the case of $\operatorname{Ag}(110)$ they contribute to $E_{\text {ads }}$, with $27 \%$ of the binding energy. $\operatorname{In} \operatorname{Ag}(111)$, the effect is the opposite as a repulsion energy of $0.61 \mathrm{eV}$ is found. We note that $E_{\text {ads }}$ is here calculated with respect to the PTCDA monolayer, and the binding strength will become even larger when calculated with respect to the molecule in gas phase due to the stabilizing formation energy of the monolayer.

TABLE V. Adsorption energies $E_{\text {ads }}$ for PTCDA on $\operatorname{Ag}(111)$, $\mathrm{Ag}(100)$, and $\mathrm{Ag}(110)$ calculated with the $\mathrm{PBE}+\mathrm{vdW}^{\text {surf }}$ method. The contributions coming from chemical (PBE) and vdW interactions after relaxing the systems are also shown.

\begin{tabular}{lccr}
\hline \hline & \multicolumn{3}{c}{$E_{\text {ads }}(\mathrm{eV})$} \\
\cline { 2 - 4 } & Total & PBE & vdW \\
\hline $\operatorname{Ag}(111)$ & -2.86 & 0.61 & -3.47 \\
$\operatorname{Ag}(100)$ & -2.93 & -0.01 & -2.92 \\
$\operatorname{Ag}(110)$ & -3.39 & -0.90 & -2.49 \\
\hline \hline
\end{tabular}


The accuracy of these results confirms the sensitivity to surface termination that the DFT+ $\mathrm{vdW}^{\text {surf }}$ scheme is able to achieve.

\section{SUMMARY AND OUTSTANDING CHALLENGES IN MODELING ADSORPTION PHENOMENA FROM FIRST PRINCIPLES}

We have presented a method that can deal with a wide range of interactions at inorganic/organic interfaces including chemical interactions, electrostatic interactions, Pauli repulsion, and vdW interactions. The noticeable improvement in the calculation of adsorption distances and energies with the DFT $+\mathrm{vdW}$ surf method indicates the importance of the inclusion of the collective effects present in the substrate for the calculation of vdW interactions, emphasizing its particular importance in the case of inorganic/organic interfaces. In principle, the method can be equally applied to any polarizable solid with any surface structure. In a more general perspective, however, the full treatment of the collective response found in the combined system (adsorbate/substrate) is an essential step in the direction of improved accuracy and increased reliability in computational studies of adsorption phenomena [34]. High-level quantum-chemistry methods or many-body methods such as the RPA for the correlation energy can be used for this purpose. Nevertheless, these approaches either perform well for one of the two subsystems, the solid or the isolated adsorbate, and not for the combined system, or their application to adsorption systems still awaits increasing computer power and more efficient implementations in order to treat larger supercells [110]. An alternative in this regard is the recently developed method, termed as DFT+MBD [111,112], which consists of an efficient dipole approximation to the RPA.

Another issue in terms of accuracy at the electronicstructure level is the self-interaction error present in semilocal xc functionals. This can lead to errors in charge transfer and electronic level alignment between the adsorbate and the substrate [34]. This issue can be solved by adding a fraction of exact exchange as done in hybrid functionals or, in a more general way, by employing orbital-dependent $\mathrm{xc}$ functionals [113]. However, these approaches are still not general and many of them increase computational cost that can become prohibitive for larger systems. The issue at hand is that the screening properties of molecules and solids are rather different and characterized by distinct parameter ranges [34], leading to the need of an accurate description of the electronic structure not only of the substrate or the adsorbate but also of their coupling.

Along with our interest in adsorption phenomena from a basic science perspective, we also aim to be able to describe and predict, from first principles, processes with technological relevance in which adsorption phenomena play an essential role (catalysis or organic electronics, for example). For this purpose, it is obvious that beyond achieving quantitative predictive level in model systems, we must also address realistic adsorption systems. Taking catalysis as an example, Sabbe and co-authors [114] mention the proper representation of the reactive surface and the treatment of coverage effects (at the electronic structure and mesoscopic level), among other aspects, as crucial. These include, for example, adsorption occurring at multifaceted surfaces or at substrates with the presence of dopant atoms or defects [114]. The addition of first-principles thermodynamic considerations must also be taken into account as thermal effects can lead to restructured surfaces, kinetic effects on adatoms, and vibrational effects on larger (and more flexible) adsorbed molecules.

In summary, the development of methods that are able to give a balanced description of adsorption phenomena and treat realistic adsorption systems is still experiencing its early phases. Among these methods, we find that the $\mathrm{DFT}+\mathrm{vdW}^{\text {surf }}$ method is a reasonable option for the accurate treatment of adsorption problems with particular advantage in the calculation of HIOS due to its efficiency and affordability in terms of computational time.

\section{ACKNOWLEDGMENTS}

We are grateful for support from the FP7 Marie Curie Actions of the European Commission, via the Initial Training Network SMALL (Grant No. MCITN-238804). A.T. is supported by a grant from the European Research Council (ERC Starting Grant No. VDW-CMAT).
[1] L. Kronik and N. Koch, MRS Bull. 35, 417 (2010).

[2] D. Cahen, R. Naaman, and Z. Vager, Adv. Funct. Mater. 15, 1571 (2005).

[3] S. R. Forrest and S. E. Thompson, Chem. Rev. 107, 923 (2007).

[4] S. Duhm, A. Gerlach, I. Salzmann, B. Bröker, R. L. Johnson, F. Schreiber, and N. Koch, Org. Electron. 9, 111 (2008).

[5] A. Tkatchenko, L. Romaner, O. T. Hoffmann, E. Zojer, C. Ambrosch-Draxl, and M. Scheffler, MRS Bull. 35, 435 (2010).

[6] N. Atodiresei, V. Caciuc, P. Lazić, and S. Blügel, Phys. Rev. Lett. 102, 136809 (2009).

[7] G. Mercurio, E. McNellis, I. Martin, S. Hagen, F. Leyssner, S. Soubatch, J. Meyer, M. Wolf, P. Tegeder, F. Tautz, and K. Reuter, Phys. Rev. Lett. 104, 036102 (2010).

[8] D. Stradi, S. Barja, C. Díaz, M. Garnica, B. Borca, J. J. Hinarejos, D. Sánchez-Portal, M. Alcamí, A. Arnau,
A. L. Vázquez de Parga, R. Miranda, and F. Martín, Phys. Rev. Lett. 106, 186102 (2011).

[9] T. Olsen, J. Yan, J. J. Mortensen, and K. S. Thygesen, Phys. Rev. Lett. 107, 156401 (2011).

[10] E. McNellis, Ph.D. thesis, Fritz-Haber-Institut der MPG, 2010.

[11] P. Lazić, V. Caciuc, N. Atodiresei, M. Callsen, and S. Blügel, J. Phys.: Condens. Matter 26, 263001 (2014).

[12] S. Grimme, J. Antony, S. Ehrlich, and H. Krieg, J. Chem. Phys. 132, 154104 (2010).

[13] K. Lee, E. D. Murray, L. Kong, B. I. Lundqvist, and D. C. Langreth, Phys. Rev. B 82, 081101 (2010).

[14] J. Klimeš, D. R. Bowler, and A. Michaelides, J. Phys.: Condens. Matter 22, 022201 (2010).

[15] F. O. Kannemann and A. D. Becke, J. Chem. Theory Comput. 6, 1081 (2010). 
[16] S. N. Steinmann and C. Corminboeuf, J. Chem. Theory and Comput. 7, 3567 (2011).

[17] A. Tkatchenko and M. Scheffler, Phys. Rev. Lett. 102, 073005 (2009).

[18] J. Klimeš, D. R. Bowler, and A. Michaelides, J. Chem. Phys. 137, 120901 (2012).

[19] M. Rohlfing and T. Bredow, Phys. Rev. Lett. 101, 266106 (2008).

[20] M. Rohlfing, R. Temirov, and F. S. Tautz, Phys. Rev. B 76, 115421 (2007).

[21] L. Romaner, D. Nabok, P. Puschnig, E. Zojer, and C. Ambrosch-Draxl, New J. Phys. 11, 053010 (2009).

[22] V. G. Ruiz, W. Liu, E. Zojer, M. Scheffler, and A. Tkatchenko, Phys. Rev. Lett. 108, 146103 (2012).

[23] M.-T. Nguyen, C. A. Pignedoli, M. Treier, R. Fasel, and D. Passerone, Phys. Chem. Chem. Phys. 12, 992 (2010).

[24] J. Lüder, B. Sanyal, O. Eriksson, C. Puglia, and B. Brena, Phys. Rev. B 89, 045416 (2014).

[25] E. M. Lifshitz, ZhETF 29, 94 (1956) [Sov. Phys.-JETP 2, 73 (1956)].

[26] E. Zaremba and W. Kohn, Phys. Rev. B 13, 2270 (1976).

[27] W. Liu, J. Carrasco, B. Santra, A. Michaelides, M. Scheffler, and A. Tkatchenko, Phys. Rev. B 86, 245405 (2012).

[28] J. van Ruitenbeek, Nat. Mater. 11, 834 (2012).

[29] W. Liu, V. G. Ruiz, G.-X. Zhang, B. Santra, X. Ren, M. Scheffler, and A. Tkatchenko, New J. Phys. 15, 053046 (2013).

[30] C. Bürker, N. Ferri, A. Tkatchenko, A. Gerlach, J. Niederhausen, T. Hosokai, S. Duhm, J. Zegenhagen, N. Koch, and F. Schreiber, Phys. Rev. B 87, 165443 (2013).

[31] G. Mercurio, R. J. Maurer, W. Liu, S. Hagen, F. Leyssner, P. Tegeder, J. Meyer, A. Tkatchenko, S. Soubatch, K. Reuter, and F. S. Tautz, Phys. Rev. B 88, 035421 (2013).

[32] B. Schuler, W. Liu, A. Tkatchenko, N. Moll, G. Meyer, A. Mistry, D. Fox, and L. Gross, Phys. Rev. Lett. 111, 106103 (2013).

[33] W. Liu, S. N. Filimonov, J. Carrasco, and A. Tkatchenko, Nat. Commun. 4, 2569 (2013).

[34] W. Liu, A. Tkatchenko, and M. Scheffler, Acc. Chem. Res. 47, 3369 (2014).

[35] J. Camarillo-Cisneros,W. Liu, and A. Tkatchenko, Phys. Rev. Lett. 115, 086101 (2015).

[36] D. Egger, V. G. Ruiz, W. A. Saidi, T. Bučko, A. Tkatchenko, and E. Zojer, J. Phys. Chem. C 117, 3055 (2013).

[37] G. Vidali, G. Ihm, H.-Y. Kim, and M. W. Cole, Surf. Sci. Rep. 12, 135 (1991)

[38] R. D. Diehl, T. Seyller, M. Caragiu, G. S. Leatherman, N. Ferralis, K. Pussi, P. Kaukasoina, and M. Lindroos, J. Phys.: Condens. Matter 16, S2839 (2004).

[39] L. W. Bruch, R. D. Diehl, and J. A. Venables, Rev. Mod. Phys. 79, 1381 (2007).

[40] L. W. Bruch, M. W. Cole, and E. Zaremba, Physical Adsorption: Forces and Phenomena (Oxford University Press, Oxford, 1997).

[41] J. L. Da Silva, C. Stampfl, and M. Scheffler, Phys. Rev. Lett. 90, 066104 (2003).

[42] J. L. Da Silva, C. Stampfl, and M. Scheffler, Phys. Rev. B 72, 075424 (2005).

[43] D.-L. Chen, W. A. Al-Saidi, and J. K. Johnson, Phys. Rev. B 84, 241405(R) (2011); J. Phys.: Condens. Matter 24, 424211 (2012).
[44] O. Bauer, G. Mercurio, M. Willenbockel, W. Reckien, C. H. Schmitz, B. Fiedler, S. Soubatch, T. Bredow, F. Tautz, and M. Sokolowski, Phys. Rev. B 86, 235431 (2012).

[45] G. Mercurio, O. Bauer, M. Willenbockel, N. Fairley, W. Reckien, C. H. Schmitz, B. Fiedler, S. Soubatch, T. Bredow, M. Sokolowski, and F. Tautz, Phys. Rev. B 87, 045421 (2013).

[46] H. C. Longuet-Higgins, Discuss. Faraday Soc. 40, 7 (1965).

[47] J. F. Dobson, Topics in Condensed Matter Physics (Nova, New York, 1994), p. 121.

[48] H. B. G. Casimir and B. Polder, Phys. Rev. 73, 360 (1948).

[49] F. London, Z. Phys. Chem. (Leipzig) B11, 222 (1930).

[50] J. F. Dobson, in Time-Dependent Density Functional Theory, Lecture Notes in Physics 706, edited by M. A. L. M. et al. (Springer, Berlin, 2006), p. 443.

[51] A. Tkatchenko, A. Ambrosetti, and R. A. DiStasio Jr., J. Chem. Phys. 138, 074106 (2013).

[52] R. A. DiStasio Jr., V. V. Gobre, and A. Tkatchenko, J. Phys.: Condens. Matter 26, 213202 (2014).

[53] S. Grimme, J. Comput. Chem. 25, 1463 (2004).

[54] S. Grimme, J. Comput. Chem. 27, 1787 (2006).

[55] B. N. J. Persson and E. Zaremba, Phys. Rev. B 30, 5669 (1984).

[56] F. London and M. Polanyi, Naturwissenschaften 18, 1099 (1930).

[57] J. F. Dobson and T. Gould, J. Phys.: Condens. Matter 24, 073201 (2012).

[58] M. Polanyi, Trans. Faraday Soc. 28, 316 (1932).

[59] M. Polanyi, Science 141, 1010 (1963).

[60] H. C. Hamaker, Physica (Amsterdam) 4, 1058 (1937).

[61] M. W. Cole, H.-Y. Kim, and M. Liebrecht, J. Chem. Phys. 137, 194316 (2012).

[62] S. H. Patil, K. T. Tang, and J. P. Toennies, J. Chem. Phys. 116, 8118 (2002).

[63] K. T. Tang and M. Karplus, Phys. Rev. 171, 70 (1968)

[64] K. T. Tang, Phys. Rev. 177, 108 (1969).

[65] X. Chu and A. Dalgarno, J. Chem. Phys. 121, 4083 (2004).

[66] G. Zhang, A. Tkatchenko, J. Paier, H. Appel, and M. Scheffler, Phys. Rev. Lett. 107, 245501 (2011).

[67] W. S. M. Werner, K. Glantschnig, and C. Ambrosch-Draxl, J. Phys. Chem. Ref. Data 38, 1013 (2009).

[68] D. L. Windt, W. C. Cash, Jr., M. Scott, P. Arendt, B. Newman, R. F. Fisher, and A. B. Swartzlander, Appl. Opt. 27, 246 (1988).

[69] W. S. Choi, S. S. A. Seo, K. W. Kim, T. W. Noh, M. Y. Kim, and S. Shin, Phys. Rev. B 74, 205117 (2006).

[70] H. W. King, Crystal Structures and Lattice Parameters of Allotropes of the Elements, in CRC Handbook of Chemistry and Physics, 95th ed., edited by W. M. Haynes et al. (CRC Press, Boca Raton, FL, 2014).

[71] F. L. Hirshfeld, Theor. Chim. Acta 44, 129 (1977).

[72] E. R. Johnson and A. D. Becke, J. Chem. Phys. 123, 024101 (2005).

[73] T. Brink, J. S. Murray, and P. Politzer, J. Chem. Phys. 98, 4305 (1993).

[74] P. Jurečka, J. Šponer, J. Černý, and P. Hobza, Phys. Chem. Chem. Phys. 8, 1985 (2006).

[75] N. Marom, A. Tkatchenko, M. Rossi, V. V. Gobre, O. Hod, M. Scheffler, and L. Kronik, J. Chem. Theory Comput. 7, 3944 (2011).

[76] V. Blum, R. Gehrke, F. Hanke, P. Havu, V. Havu, X. Ren, K. Reuter, and M. Scheffler, Comput. Phys. Commun. 180, 2175 (2009). 
[77] J. H. van Lenthe, S. Faas, and J. G. Snijders, Chem. Phys. Lett. 328, 107 (2000).

[78] J. P. Perdew, K. Burke, and M. Ernzerhof, Phys. Rev. Lett. 77, 3865 (1996).

[79] C. Wagner, N. Fournier, F. S. Tautz, and R. Temirov, Phys. Rev. Lett. 109, 076102 (2012).

[80] H. J. Monkhorst and J. D. Pack, Phys. Rev. B 13, 5188 (1976).

[81] A. Hauschild, K. Karki, B. Cowie, M. Rohlfing, F. Tautz, and M. Sokolowski, Phys. Rev. Lett. 94, 036106 (2005).

[82] K. Glöckler, C. Seidel, A. Soukopp, M. Sokolowski, E. Umbach, M. Bohringer, R. Berndt, and W.-D. Schneider, Surf. Sci. 405, 1 (1998).

[83] J. Ikonomov, O. Bauer, and M. Sokolowski, Surf. Sci. 602, 2061 (2008).

[84] T. Seyller, M. Caragiu, R. D. Diehl, P. Kaukasoina, and M. Lindroos, Chem. Phys. Lett. 291, 567 (1998).

[85] M. Caragiu, T. Seyller, and R. D. Diehl, Surf. Sci. 539, 165 (2003).

[86] V. Pouthier, C. Ramseyer, C. Girardet, V. Diercks, R. Halmer, R. David, and P. Zeppenfeld, Phys. Rev. B 57, 13149 (1998).

[87] J. F. Zhu, H. Ellmer, H. Malissa, T. Brandstetter, D. Semrad, and P. Zeppenfeld, Phys. Rev. B 68, 045406 (2003).

[88] M. Caragiu, T. Seyller, and R. D. Diehl, Phys. Rev. B 66, 195411 (2002).

[89] G. Hilgers, M. Potthoff, and N. Müller, Surf. Sci. 322, 207 (1995).

[90] W. Widdra, P. Trischberger, W. Frieß, D. Menzel, S. H. Payne, and H. J. Kreuzer, Phys. Rev. B 57, 4111 (1998).

[91] T. Seyller, M. Caragiu, R. D. Diehl, P. Kaukasoina, and M. Lindroos, Phys. Rev. B 60, 11084 (1999).

[92] L. W. Bruch, A. P. Graham, and J. P. Toennies, Mol. Phys. 95, 579 (1998)

[93] B. Hall, D. L. Mills, P. Zeppenfeld, K. Kern, U. Becher, and G. Comsa, Phys. Rev. B 40, 6326 (1989).

[94] J. Braun, D. Fuhrmann, A. Siber, B. Gumhalter, and C. Wöll, Phys. Rev. Lett. 80, 125 (1998).
[95] P. Zeppenfeld, M. Büchel, R. David, G. Comsa, C. Ramseyer, and C. Girardet, Phys. Rev. B 50, 14667 (1994).

[96] C. Ramseyer, V. Pouthier, C. Girardet, P. Zeppenfeld, M. Büchel, V. Diercks, and G. Comsa, Phys. Rev. B 55, 13203 (1997).

[97] J. Ellis, A. P. Graham, and J. P. Toennies, Phys. Rev. Lett. 82, 5072 (1999).

[98] K. D. Gibson and S. J. Sibener, J. Chem. Phys. 88, 7862 (1988).

[99] J. Carrasco, W. Liu, A. Michaelides, and A. Tkatchenko, J. Chem. Phys. 140, 084704 (2014).

[100] R. J. Maurer, V. G. Ruiz, and A. Tkatchenko, J. Chem. Phys. 143, 102808 (2015).

[101] S. K. M. Henze, O. Bauer, T.-L. Lee, M. Sokolowski, and F. Tautz, Surf. Sci. 601, 1566 (2007).

[102] F. S. Tautz, Prog. Surf. Sci. 82, 479 (2007).

[103] J. Ziroff, P. Gold, A. Bendounan, F. Forster, and F. Reinert, Surf. Sci. 603, 354 (2009).

[104] A. Hauschild, R. Temirov, S. Soubatch, O. Bauer, A. Schöll, B. Cowie, and T.-L. Lee, Phys. Rev. B 81, 125432 (2010).

[105] S. Stremlau, Ph.D. thesis, Freie Universität Berlin, 2015.

[106] K. Momma and F. Izumi, J. Appl. Crystallogr. 44, 1272 (2011).

[107] W. A. Al-Saidi, H. Feng, and K. A. Fichthorn, Nano Lett. 12, 997 (2012).

[108] A. Kraft, R. Temirov, S. K. M. Henze, S. Soubatch, M. Rohlfing, and F. S. Tautz, Phys. Rev. B 74, 041402(R) (2006).

[109] M. Böhringer, W.-D. Schneider, K. Glöckler, E. Umbach, and R. Berndt, Surf. Sci. 419, L95 (1998).

[110] X. Ren, P. Rinke, C. Joas, and M. Scheffler, J. Mater. Sci. 47, 7447 (2012).

[111] A. Tkatchenko, R. A. DiStasio Jr., R. Car, and M. Scheffler, Phys. Rev. Lett. 108, 236402 (2012).

[112] A. Ambrosetti, A. M. Reilly, R. A. DiStasio Jr., and A. Tkatchenko, J. Chem. Phys. 140, 18A508 (2014).

[113] S. Kümmel and L. Kronik, Rev. Mod. Phys. 80, 3 (2006).

[114] M. K. Sabbe, M.-F. Reyniers, and K. Reuter, Catal. Sci. Technol. 2, 2010 (2012). 